\title{
ON THE DIMENSION OF CERTAIN GRADED LIE ALGEBRAS ARISING IN GEOMETRIC INTEGRATION OF DIFFERENTIAL EQUATIONS
}

\author{
ARIEH ISERLES AND ANTONELLA ZANNA
}

\begin{abstract}
Many discretization methods for differential equations that evolve in Lie groups and homogeneous spaces advance the solution in the underlying Lie algebra. The main expense of computation is the calculation of commutators, a task that can be made significantly cheaper by the introduction of appropriate bases of function values and by the exploitation of redundancies inherent in a Lie-algebraic structure by means of graded spaces. In many Lie groups of practical interest a convenient alternative to the exponential map is a Cayley transformation, and the subject of this paper is the investigation of graded algebras that occur in this context. To this end we introduce a new concept, a hierarchical algebra, a Lie algebra equipped with a countable number of $m$-nary multilinear operations which display alternating symmetry and a 'hierarchy condition'. We present explicit formulae for the dimension of graded subspaces of free hierarchical algebras and an algorithm for the construction of their basis. The paper is concluded by reviewing a number of applications of our results to numerical methods in a Lie-algebraic setting.
\end{abstract}

\section{Graded algebras and Lie-group methods}

\subsection{Geometric integration and general Lie-group solvers}

An increasing amount of attention has been paid in recent years to the discretization of differential equations that evolve on smooth manifolds. The main reason is that the invariants and conservation laws of a differential system can be phrased by restricting the configuration space to a manifold. Discretization methods that respect the manifold structure are an important example of geometric integrators, computational methods that preserve the underlying geometry and qualitative attributes of the differential system [1]. Perhaps the most ubiquitous (and arguably most important) type of manifold that occurs in practical applications is a Lie group. Thus, numerous differential equations of practical interest evolve in Lie groups, and the conservation of this feature under discretization is often valuable and sometimes crucial.

- Equations of classical mechanics, robotics and control theory often evolve on the special orthogonal group $\mathrm{SO}_{n}(\mathbb{R})$. This is also the case with several processes of interest in numerical algebra and computational dynamics.

- Many equations in special relativity evolve in the Lorenz group $\mathrm{SO}_{3,1}(\mathbb{R})$. 
- Much of quantum theory can be formulated as evolution in the unitary group $\mathrm{U}_{n}(\mathbb{C})$.

- Conservation of volume, ubiquitous in equations of incompressible flow, corresponds to evolution in the special linear group $\mathrm{SL}_{n}(\mathbb{R})$.

Detailed references can be found in [1]. The central role of Lie groups in geometric integration is underscored by the fact that, as long as we can discretize a flow evolving in a Lie group ( $G$, say), we can also discretize differential systems evolving in an arbitrary homogeneous space which is acted upon by $G$ [18]. Thus, for example, a numerical scheme that is guaranteed to respect an $\mathrm{O}_{n}(\mathbb{R})$ structure can be easily adapted to evolve on a sphere (say, a torus), an isospectral manifold, or a Stiefel or Grassmann manifold.

Below, we survey briefly a number of state-of-the-art Lie-group integrators. A major computational bottleneck of such algorithms is that they require substantial linear-algebra computations. This important issue has been recently addressed in [17], employing techniques of graded Lie algebras, and this has led to very substantial savings. The approach of [17], however, cannot be applied to the recently introduced Lie-group integrators based on the Cayley transform $[\mathbf{6}, \mathbf{7}, \mathbf{1 1}, \mathbf{1 3}]$. The purpose of this paper is to establish a mathematical framework which uses a different type of graded algebras to extend the benefits of [17] to Lie-group integrators based on the Cayley transform. As in the case of [17], the savings implicit in our approach become increasingly apparent for high-order methods. Thus, a fourth-order Cayley expansion from [11] requires four matrix products per step in either a naive or a graded approach. In the case of a sixth-order method, the number of matrix products drops from 23 to 17 with the use of graded algebras, and the savings become considerably more pronounced for higher orders.

Although exceptions exist $[\mathbf{5}, \mathbf{4}, \mathbf{1 5}, \mathbf{2 0}]$, most Lie-group integrators follow a set pattern. A flow in a finite-dimensional Lie group $G$ can be always written in the form

$$
Y^{\prime}=A(t, Y) Y, \quad t \geqslant 0, \quad Y\left(t_{0}\right)=Y_{0} \in G,
$$

where $A$ is an adequately smooth map from $\left[t_{0}, \infty\right) \times G$ to $\mathfrak{g}$, the Lie algebra corresponding to $G$. Representing $Y(t)=\exp [\Omega(t)] Y_{0}$, we can replace equation (1) with an equation evolving in the Lie algebra, namely the so-called 'dexpinv equation'

$$
\Omega^{\prime}=\operatorname{dexp}_{\Omega}^{-1} A\left(t, \mathrm{e}^{\Omega} Y_{0}\right)=\sum_{k=0}^{\infty} \frac{B_{k}}{k !} \operatorname{ad}_{\Omega}^{k} A\left(t, \mathrm{e}^{\Omega} Y_{0}\right), \quad t \geqslant t_{0}, \quad \Omega\left(t_{0}\right)=O,
$$

where $\left\{B_{k}\right\}_{k \geqslant 0}$ are Bernoulli numbers, while ad $X$ is the adjoint operator in $\mathfrak{g}$,

$$
\operatorname{ad}_{X}^{m}=\overbrace{[X,[X, \ldots,[X, Y] \cdots]]}^{m \text { times }}, \quad m \geqslant 0 .
$$

Instead of solving (1) directly, we apply a numerical scheme to (2) and translate the outcome to $G$ with the exponential map. The reasoning underlying this approach is that, unlike nontrivial Lie groups, Lie algebras are linear spaces. Discretization methods that restrict themselves, as most numerical algorithms do, to linear operations and commutation, are guaranteed (within machine accuracy) to evolve in the Lie algebra! Although an implementation of the exponential map calls for much care and algorithmic dexterity [2], this procedure nonetheless provides a convenient avenue toward Lie-group solvers.

Perhaps the most versatile example of a Lie-group solver that follows the above pattern is provided by Runge-Kutta-Munthe-Kaas methods [16]. The main idea is to apply a $v$-stage 
Runge-Kutta method

$$
\begin{array}{c|cccc}
c_{1} & a_{1,1} & a_{1,2} & \cdots & a_{1, v} \\
c_{2} & a_{2,1} & a_{2,2} & \cdots & a_{2, v} \\
\vdots & \vdots & \vdots & & \vdots \\
c_{v} & a_{v, 1} & a_{v, 2} & \cdots & a_{v, v} \\
\hline & b_{1} & b_{2} & \cdots & b_{v}
\end{array}
$$

(cf. [10] for Runge-Kutta formalism) to the Lie-algebraic equation (2). Denoting the approximate value of the solution of (1) at time $t_{N}$ by $Y_{N}$ and letting $h=t_{N+1}-t_{N}$, we thus obtain a scheme of the form

$$
\left.\begin{array}{rl}
\Phi_{m} & =h \sum_{l=1}^{v} a_{m, l} K_{l}, \\
K_{m} & =\operatorname{dexp}_{\Phi_{m}}^{-1} A\left(t_{N}+c_{m} h, \mathrm{e}^{\Phi_{m}} Y_{N}\right),
\end{array}\right\} m=1,2, \ldots, v,
$$

It is of the same order of precision as the original Runge-Kutta method, but it is guaranteed to evolve on $G$.

Another important example of a Lie-group solver is the Magnus expansion which, for linear equations $Y^{\prime}=A(t) Y$, reads

$$
\begin{aligned}
\Omega(t)= & \int_{t_{0}}^{t} A(\xi) \mathrm{d} \xi-\frac{1}{2} \int_{t_{0}}^{t} \int_{t_{0}}^{\xi_{1}}\left[A\left(\xi_{2}\right), A\left(\xi_{1}\right)\right] \mathrm{d} \xi_{2} \mathrm{~d} \xi_{1} \\
& +\frac{1}{4} \int_{t_{0}}^{t} \int_{t_{0}}^{\xi_{1}} \int_{t_{0}}^{\xi_{2}}\left[\left[A\left(\xi_{3}\right), A\left(\xi_{2}\right)\right], A\left(\xi_{1}\right)\right] \mathrm{d} \xi_{3} \mathrm{~d} \xi_{2} \mathrm{~d} \xi_{1} \\
& +\frac{1}{2} \int_{t_{0}}^{t} \int_{t_{0}}^{\xi_{1}} \int_{t_{0}}^{\xi_{1}}\left[A\left(\xi_{3}\right),\left[A\left(\xi_{2}\right), A\left(\xi_{1}\right)\right]\right] \mathrm{d} \xi_{3} \mathrm{~d} \xi_{2} \mathrm{~d} \xi_{1}+\cdots
\end{aligned}
$$

originally introduced by Wilhelm Magnus [14]. Recently it has been cast by Iserles and Nørsett into a form amenable to numerical treatment [12]. This has included recursive generation of expansion terms, and an effective treatment of multivariate integrals over polytopes by numerical quadrature. A nonlinear version of the Magnus expansion, using collocation, is due to Zanna [21].

Yet another example of a Lie-group solver based on reducing the problem to a Lie algebra is the Fer expansion [8]

$$
Y(t)=\mathrm{e}^{\Omega^{[0]}(t)} \mathrm{e}^{\Omega^{[1]}(t)} \cdots Y_{0} .
$$

In the linear case the Lie-algebra functions $\Omega^{[i]}$ can be obtained recursively [9]; in addition, this procedure can be generalized to nonlinear equations [21].

\subsection{Quadratic Lie groups and the Cayley transform}

Runge-Kutta-Munthe-Kaas schemes, as well as Magnus and Fer expansions, can be applied to all finite-dimensional Lie groups, regardless of their structure. This is their strength, yet it also represents a potential shortcoming. Many Lie groups possess structures that can be exploited to construct more effective discretization methods. A case in point comprises 
quadratic Lie groups [11]: given $J \in \mathrm{GL}_{n}(\mathbb{R})$, the set of $n \times n$ real nonsingular matrices, we let

$$
G=\left\{Y \in \mathrm{GL}_{n}(\mathbb{R}): Y J Y^{\mathrm{T}}=J\right\} .
$$

Note that $\mathrm{SO}_{n}(\mathbb{R}), \mathrm{Sp}_{n}$ and $\mathrm{SO}_{3,1}(\mathbb{R})$ are all either quadratic Lie groups or connected components thereof, while $\mathrm{U}_{n}(\mathbb{C})$ and other complex quadratic groups can be obtained by replacing equation (5) with $\left\{Y \in \mathrm{GL}_{n}(\mathbb{C}): Y J Y^{\mathrm{H}}=J\right\}$. Thus, quadratic Lie groups account for four out of our five examples of matrix Lie groups that are relevant in applications. This emphasises the importance of this construct, and justifies special attention being paid to quadratic Lie-group methods.

The main structural feature of quadratic Lie groups that can be exploited in the context of Lie-group solvers is that we do not need to use (or approximate) the matrix exponential in order to map $G$ to its Lie algebra

$$
\mathfrak{g}=\left\{X \in \mathfrak{g l}_{n}(\mathbb{R}): X J+J X^{\mathrm{T}}=O\right\} .
$$

An alternative is to employ the Cayley transform, letting $Y(t)=$ cay $[\Omega(t)] Y_{0}, t \geqslant t_{0}$, where

$$
\text { cay } X=\left(I-\frac{1}{2} X\right)^{-1}\left(I+\frac{1}{2} X\right) \text {. }
$$

Thus, the evaluation of the exponential — a costly procedure-is no longer required, and can be replaced by the much cheaper matrix inversion. Moreover, in place of the dexpinv equation (2) one obtains the considerably simpler dcayinv equation

$$
\Omega^{\prime}=\operatorname{dcay}_{\Omega}^{-1} A\left(t, \operatorname{cay}[\Omega] Y_{0}\right)=A-\frac{1}{2}[\Omega, A]-\frac{1}{4} \Omega A \Omega, \quad t \geqslant t_{0}, \quad \Omega\left(t_{0}\right)=O .
$$

This has been recognized in [13] and further exploited in [7, 6]. Recently, Iserles introduced a Cayley-transform equivalent of a Magnus expansion [11]. Specifically, for linear equations $Y^{\prime}=A(t) Y$, the function $\Omega$ is expanded in terms of integrals, commutators and symmetric products $P Q R+R Q P$,

$$
\begin{aligned}
\Omega(t)= & \int_{t_{0}}^{t} A(\xi) \mathrm{d} \xi-\frac{1}{2} \int_{t_{0}}^{t} \int_{t_{0}}^{\xi_{1}}\left[A\left(\xi_{2}\right), A\left(\xi_{1}\right)\right] \mathrm{d} \xi_{2} \mathrm{~d} \xi_{1} \\
& +\frac{1}{4} \int_{t_{0}}^{t} \int_{t_{0}}^{\xi_{1}} \int_{t_{0}}^{\xi_{2}}\left[\left[A\left(\xi_{3}\right), A\left(\xi_{2}\right)\right], A\left(\xi_{1}\right)\right] \mathrm{d} \xi_{3} \mathrm{~d} \xi_{2} \mathrm{~d} \xi_{1} \\
& -\frac{1}{4} \int_{t_{0}}^{t} \int_{t_{0}}^{\xi_{1}} \int_{t_{0}}^{\xi_{1}} A\left(\xi_{2}\right) A\left(\xi_{1}\right) A\left(\xi_{3}\right) \mathrm{d} \xi_{3} \mathrm{~d} \xi_{2} \mathrm{~d} \xi_{1}+\cdots
\end{aligned}
$$

The Cayley expansion (9) has a number of advantages compared to the Magnus expansion (4): the number of terms with any given number of integrals is smaller, the radius of convergence is more generous and, as we have already mentioned, there is no need whatsoever to evaluate a matrix exponential. The general form of equation (9) has been investigated in great detail in [11] by identifying each expansion term with a bicolour rooted tree, whereby expansion coefficients can be generated recursively and counted by combinatorial arguments.

\subsection{Quadrature and graded Lie algebras}

Practical implementation of a Magnus or Cayley expansion requires the truncation of the infinite series and an approximation of integrals by quadrature. On the face of it, the latter task is likely to be prohibitively expensive, since each expansion term requires an integration in a different multivariate polytope. Using a traditional cubature method would 
have required a truly enormous number of function evaluations [3]. Fortunately, the structure of the integrals featuring in either equation (4) or equation (9) can be exploited to very good effect, and this leads to a remarkably efficient quadrature [12]. Assuming without loss of generality that $t_{0}=0$ and advancing the integration with time step $h>0$, all such integrals are of the form

$$
\mathcal{I}(h)=\int_{h \S} \mathcal{L}\left(A\left(\xi_{1}\right), A\left(\xi_{2}\right), \ldots, A\left(\xi_{r}\right)\right) \mathrm{d} \xi_{r} \mathrm{~d} \xi_{r-1} \cdots \mathrm{d} \xi_{1},
$$

where $\mathcal{L}$ is a multilinear form, while $s \subset \mathbb{R}$ is a polytope,

$$
\S=\left\{\xi \in \mathbb{R}^{r}: 0 \leqslant \xi_{i} \leqslant \xi_{q_{i}}, i=1,2, \ldots, r\right\}
$$

with $\xi_{0}=1$ and $q_{i} \leqslant \max \{0, i-1\}$, for $i=1,2, \ldots, r$. Let $c_{1}, c_{2}, \ldots, c_{\nu}$ be arbitrary distinct quadrature points in $[0,1]$, and let $A_{k}=A\left(c_{k} h\right)$, for $k=1,2, \ldots, v$. We approximate $\mathcal{I}(h)$ by the quadrature formula

$$
\mathcal{Q}(h)=h^{r} \sum_{k_{1}=1}^{v} \sum_{k_{2}=1}^{v} \cdots \sum_{k_{r}=1}^{v} b_{\boldsymbol{k}} \mathcal{L}\left(A_{k_{1}}, A_{k_{2}}, \ldots, A_{k_{r}}\right),
$$

where the weights $b_{\boldsymbol{k}}$ can be constructed explicitly by integrating cardinal Lagrange interpolation polynomials $[\mathbf{1 1}, \mathbf{1 2}]$. The crucial observation is that the order of accuracy of equation (10) is precisely that of the univariate Gaussian quadrature in $[0,1]$ (with constant weight function) using the nodes $c_{1}, c_{2}, \ldots, c_{v}$. In particular, letting the nodes equal the zeros of the $v$ th Legendre polynomial, shifted to $[0,1]$, we obtain

$$
\mathcal{Q}(h)=\mathcal{I}(h)+\mathcal{O}\left(h^{2 v+1}\right)
$$

for all sufficiently smooth matrix functions $A$. Note that we use the very same $v$ function values in all the many integrals occurring in the expansion!

Quadrature formulae (10) require a very small number of function evaluations to compute a Magnus or a Cayley expansion, but this is traded off for a substantial number of matrix products: the number of combinations increases very fast as a function or $r$ and $v$, and each such combination requires the computation of a multilinear form $\mathcal{L}\left(A_{k_{1}}, A_{k_{2}}, \ldots, A_{k_{r}}\right)$. Fortunately, very significant savings in linear algebra can be obtained by exploiting three mechanisms. Firstly, combinations in one multilinear form might be identical (up to known sign change) to different combinations of another multilinear form [12]. Secondly, expressed in a suitable basis, many combinations can be disregarded since they are of magnitude $\mathcal{O}\left(h^{q+1}\right)$ where $q$ exceeds the order of the method. Thirdly, a Lie algebra possesses a wide range of redundancies and symmetries which lead to numerous linear dependencies among values of $\mathcal{L}\left(A_{k_{1}}, A_{k_{2}}, \ldots, A_{k_{r}}\right)$ for different combinations $\boldsymbol{k}$. The last two mechanisms have been analysed in great detail by Munthe-Kaas and Owren in [17] in the context of Magnus expansions. The main purpose of the present paper is to extend their analysis to the realm of Cayley expansions and other algorithms specific to quadratic Lie groups. We note that, although our main purpose is to facilitate the computation of the Cayley expansion (9), our construction lends itself to other applications, for example Runge-Kutta-Munthe-Kaas schemes and the $\mathrm{BCH}$ formula in a Cayley setting.

The first crucial idea in [17] is to express the function values $\left\{A_{1}, A_{2}, \ldots, A_{v}\right\}$ using a different basis, which renders the order of each term more transparent. Specifically, we use 
the basis $\left\{B_{0}, B_{1}, \ldots, B_{v-1}\right\}$, where

$$
\sum_{l=0}^{\nu-1} \frac{1}{l !}\left(c_{k}-\frac{1}{2}\right)^{l} B_{l}=A_{k}, \quad k=1,2, \ldots, v .
$$

The quadrature formula (10) assumes the form

$$
\widetilde{\mathcal{Q}}(h)=h^{r} \sum_{l_{1}=0}^{\nu-1} \sum_{l_{2}=0}^{\nu-1} \cdots \sum_{l_{r}=0}^{\nu-1} \tilde{b}_{l} \mathcal{L}\left(B_{l_{1}}, B_{l_{2}}, \ldots, B_{l_{r}}\right),
$$

where

$$
\tilde{b}_{\boldsymbol{l}}=\frac{1}{l_{1} ! l_{2} ! \cdots l_{r} !} \int_{\delta} \prod_{j=1}^{r}\left(\xi_{j}-\frac{1}{2}\right)^{l_{j}} \mathrm{~d} \xi_{r} \mathrm{~d} \xi_{r-1} \cdots \mathrm{d} \xi_{1} .
$$

Assuming that $A \in \mathrm{C}^{\infty}$, it is trivial to verify that $B_{l}=h^{l} A^{(l)}\left(\frac{1}{2} h\right)$; therefore $B_{l}=\mathcal{O}\left(h^{l}\right)$, for $l=0,1, \ldots, v-1$. Constructing $\mathcal{L}\left(B_{l_{1}}, B_{l_{2}}, \ldots, B_{l_{r}}\right)$ from commutators and symmetric products, it follows at once that

$$
h^{r} \mathcal{L}\left(B_{l_{1}}, B_{l_{2}}, \ldots, B_{l_{r}}\right)=\mathcal{O}\left(h^{|l|+r}\right) \quad \text { where } \quad|\boldsymbol{l}|=\sum_{j=1}^{r} l_{j} .
$$

We say that such a term is of grade $|\boldsymbol{l}|+r$. Typically, $c_{1}, c_{2}, \ldots, c_{v}$ are taken as GaussLegendre points, whence equation (11) is an order- $(2 v)$ quadrature. In that case, wecan throw away with impunity all terms of grade exceeding $2 v$, and replace equation (11) by

$$
\widehat{\mathcal{Q}}(h)=h^{r} \sum_{|l| \leqslant 2 v-r} \tilde{b}_{l} \mathcal{L}\left(B_{l_{1}}, B_{l_{2}}, \ldots, B_{l_{r}}\right) .
$$

Inasmuch as this procedure gets rid of a very significant proportion of the expansion terms, further savings take place by exploiting linear dependencies in the underlying Lie algebra. In the context of 'plain' Lie algebras, the subject of [17], the two dependencygenerating mechanisms are the skew-symmetry $[Y, X]+[X, Y]=O$ and the Jacobi identity $[X,[Y, Z]]+[Y,[Z, X]]+[Z,[X, Y]]=O$, valid for all $X, Y, Z \in \mathfrak{g}$.

Given the generators $B_{0}, B_{1}, \ldots, B_{v-1}$, we assign to each $B_{l}$ a grade $\omega\left(B_{l}\right) \in \mathbb{N}$. Grades propagate naturally during the construction of a free Lie algebra: if $\omega\left(X_{i}\right)=w_{i}$, for $i=1,2$, $X_{1} \neq X_{2}$, then $\omega\left(\left[X_{1}, X_{2}\right]\right)=w_{1}+w_{2}$. Let $\mathcal{K}_{m}$ be the linear space of all words of length $m$ in a free Lie algebra, whereby $\mathfrak{g}=\bigsqcup_{m \geqslant 1}$ span $\mathcal{K}_{m}$ is a graded Lie algebra. In the case $\omega\left(B_{l}\right) \equiv 1$ the dimension of the linear space spanned by $\mathcal{K}_{m}$ is known and given by the familiar Witt formula. Moreover, it is possible to form recursively a basis of this space (for example, a Hall or a Lyndon basis). A major result of [17] is a generalisation of the Witt formula and of Hall and Lyndon bases to the case when the grades are arbitrary natural numbers. (In the present case we are interested in $w\left(B_{l}\right)=l+1$, where $l=0,1, \ldots, v-1$, but other choices of grades are of independent interest, cf. [17].) Further observing that $b_{l}=0$ for terms of even grade, Munthe-Kaas and Owren were able to reduce the number of terms in a Magnus expansion by a remarkable factor. For example, an order-10 method requires just 73 terms, instead of 1256567 in a naive implementation!

The formation of a free algebra for Lie algebra (6) allows the use of both commutators and symmetric products, while its special structure implies the existence of linear dependencies which are absent in other Lie algebras. In Section 2 we define a hierarchical algebra, an 
algebraic construct which encapsulates the special features of the Lie algebra (6), and prove that every hierarchical algebra is an extension of a Lie algebra. This is followed in Section 3 by a determination of the dimension and construction of a basis of a linear space of a given grade within the realm of freely-generated hierarchical algebras. It is interesting to note that not just the method of analysis, but also the explicit form of our formulae, are very different from the Witt and Munthe-Kaas-Owren framework. Finally, in Section 4 we present a number of examples that implement our results, not just in the context of Cayley expansions (9), but also for other computational constructs for quadratic Lie algebras.

\section{Hierarchical algebras}

Let $\mathbb{F}$ be a field of characteristic 0 . We recall that an algebra over $\mathbb{F}$ is a triple $(A,+, \circ)$, whereby $(A,+)$ is an abelian group and ' $\circ$ ' is an internal binary operation over $A$ which is linear in both arguments. In particular, a Lie algebra $\mathfrak{g}$ is an algebra whereby the binary operation ' $\circ$ ' is usually represented by a bracket $[\cdot, \cdot]$ and obeys:

1. skew-symmetry: $\forall X, Y \in \mathfrak{g}$,

$$
[X, Y]+[Y, X]=O
$$

2. Jacobi identity: $\forall X, Y, Z \in \mathfrak{g}$,

$$
[X,[Y, Z]]+[Y,[Z, X]]+[Z,[X, Y]]=O .
$$

For the time being, let $\mathfrak{g}$ represent a set, not necessarily associated with a Lie algebra. Assume that $(\mathfrak{g},+)$ is an abelian group, and let us introduce the following family of $m$-nary operations,

$$
\llbracket[, \ldots, \cdot \rrbracket_{m}: \underbrace{\mathfrak{g} \times \cdots \times \mathfrak{g}}_{m \text { copies }} \rightarrow \mathfrak{g}, \quad m=1,2, \ldots
$$

Assume that the $m$-nary operation (15) obeys

1. alternating symmetry: $\forall X_{1}, \ldots, X_{m} \in \mathfrak{g}$,

$$
\llbracket X_{1}, X_{2}, \ldots, X_{m} \rrbracket_{m}+(-1)^{m} \llbracket X_{m}, X_{m-1}, \ldots, X_{1} \rrbracket_{m}=O ;
$$

2. multilinearity: $\forall X_{1}, \ldots, X_{l-1}, X_{l+1}, \ldots X_{m}, Y, Z, \in \mathfrak{g}$ and $\forall \alpha, \beta \in \mathbb{F}$,

$$
\begin{aligned}
& \llbracket X_{1}, \ldots, X_{l-1}, \alpha Y+\beta Z, X_{l+1}, \ldots, X_{m} \rrbracket_{m} \\
= & \alpha \llbracket X_{1}, \ldots, X_{l-1}, Y, X_{l+1}, \ldots, X_{m} \rrbracket_{m}+\beta \llbracket X_{1}, \ldots, X_{l-1}, Z, X_{l+1}, \ldots, X_{m} \rrbracket_{m}
\end{aligned}
$$

for all $1 \leqslant l \leqslant m$

3. hierarchy condition: for every $n \geqslant 1$, for every index $1 \leqslant l \leqslant m$, and elements $X_{1}, \ldots, X_{l-1}, X_{l+1}, \ldots, X_{m} \in \mathfrak{g}$ and $Y_{1}, Y_{2}, \ldots, Y_{n} \in \mathfrak{g}$, then

$$
\begin{aligned}
& \llbracket X_{1}, \ldots, X_{l-1}, \llbracket Y_{1}, Y_{2}, \ldots, Y_{n} \rrbracket_{n}, X_{l+1}, \ldots, X_{m} \rrbracket_{m} \\
= & \llbracket X_{1}, \ldots, X_{l-1}, Y_{1}, \ldots, Y_{n}, X_{l+1}, \ldots, X_{m} \rrbracket_{m+n-1} \\
& -(-1)^{n} \llbracket X_{1}, \ldots, X_{l-1}, Y_{n}, \ldots, Y_{1}, X_{l+1}, \ldots, X_{m} \rrbracket_{m+n-1} .
\end{aligned}
$$

We remark that the hierarchy condition (18) links the $m$-nary product with similar products of higher order, thus establishing a hierarchy in the family of $m$-nary operations. 


\section{Graded Lie algebras}

Definition 1. A hierarchical algebra is a family $\left(\mathfrak{g},+,\left\{[\cdots]_{m}\right\}_{m=1}^{\infty}\right)$, whereby $(\mathfrak{g},+)$ is an abelian group and each $m$-nary operation obeys the alternating symmetry condition (16), the multilinearity (17) and the hierarchy condition (18).

The use of the symbol $\mathfrak{g}$, usually reserved for Lie algebras, in a hierarchical algebra setting is justified by the result below.

Theorem 1. If $\left(\mathfrak{g},+,\left\{\llbracket \cdots \rrbracket_{m}\right\}_{m=1}^{\infty}\right)$ is a hierarchical algebra, then $\left(\mathfrak{g},+, \llbracket \cdots \rrbracket_{2}\right)$ is a Lie algebra.

Proof. It is sufficient to prove that the binary product $[\cdots \cdots]_{2}$ obeys the Jacobi identity, since skew-symmetry is an immediate consequence of the alternating symmetry condition (16).

Consider three arbitrary elements $X, Y, Z \in \mathfrak{g}$. Because of the hierarchy condition,

$$
\begin{aligned}
\llbracket X, \llbracket Y, Z \rrbracket_{2} \rrbracket_{2} & =\llbracket X, Y, Z \rrbracket_{3}-\llbracket\left[X, Z, Y \rrbracket_{3},\right. \\
\llbracket Y, \llbracket Z, X \rrbracket_{2} \rrbracket_{2} & =\llbracket Y, Z, X \rrbracket_{3}-\llbracket Y, X, Z \rrbracket_{3}, \\
\llbracket\left[Z, \llbracket X, Y \rrbracket_{2} \rrbracket_{2}\right. & =\llbracket Z, X, Y \rrbracket_{3}-\llbracket Z, Y, X \rrbracket_{3} .
\end{aligned}
$$

Furthermore, the alternating symmetry condition implies that

$$
\llbracket X_{1}, X_{2}, X_{3} \rrbracket_{3}=\llbracket X_{3}, X_{2}, X_{1} \rrbracket_{3}
$$

for every $X_{1}, X_{2}, X_{3} \in \mathfrak{g}$. Hence we deduce that

$$
\begin{aligned}
\llbracket X, \llbracket Y, Z \rrbracket_{2} \rrbracket_{2}+\llbracket Y, \llbracket Z, X \rrbracket_{2} \rrbracket_{2} & =\llbracket X, Y, Z \rrbracket_{3}-\llbracket X, Z, Y \rrbracket_{3}+\llbracket Y, Z, X \rrbracket_{3}-\llbracket Y, X, Z \rrbracket_{3} \\
& =\llbracket X, Y, Z \rrbracket_{3}-\llbracket Y, X, Z \rrbracket_{3}=-\llbracket Z, \llbracket X, Y \rrbracket_{2} \rrbracket_{2},
\end{aligned}
$$

from which the Jacobi identity follows.

Theorem 2. Every quadratic matrix Lie algebra is a hierarchical algebra.

Proof. Consider the operation

$$
\llbracket X_{1}, \ldots, X_{m} \rrbracket_{m}=X_{1} X_{2} \cdots X_{m}-(-1)^{m} X_{m} X_{m-1} \cdots X_{1}
$$

with the usual matrix-matrix multiplication. Then, for every $m \geqslant 1$,

$$
\begin{aligned}
& \llbracket X_{1}, \ldots, X_{m} \rrbracket_{m}+(-1)^{m} \llbracket X_{m}, \ldots, X_{1} \rrbracket_{m} \\
= & X_{1} X_{2} \cdots X_{m}-(-1)^{m} X_{m} X_{m-1} \cdots X_{1}+(-1)^{m}\left(X_{m} \cdots X_{1}-(-1)^{m} X_{1} \cdots X_{m}\right) \\
= & X_{1} X_{2} \cdots X_{m}-(-1)^{m} X_{m} X_{m-1} \cdots X_{1}+(-1)^{m} X_{m} \cdots X_{1}-X_{1} \cdots X_{m}=O,
\end{aligned}
$$

hence alternating symmetry. The multilinearity of the above $m$-nary operation is immediate.

Next, let us prove the hierarchy condition. To this end, given $l \in\{1,2, \ldots, m\}$ and the elements $X_{1}, \ldots, X_{l-1}, X_{l+1}, \ldots X_{m}$ and $Y_{1}, \ldots Y_{n} \in \mathfrak{g}$, we have

$$
\begin{aligned}
& \llbracket X_{1}, \ldots, X_{l-1}, \llbracket Y_{1}, \ldots, Y_{n} \rrbracket_{n}, X_{l+1}, \ldots, X_{m} \rrbracket_{m} \\
= & X_{1} \cdots X_{l-1} Y_{1} \cdots Y_{n} X_{l+1} \cdots X_{m}-(-1)^{n} X_{1} \cdots X_{l-1} Y_{n} \cdots Y_{1} X_{l+1} \cdots X_{m} \\
& -(-1)^{m} X_{m} \cdots X_{l+1} Y_{1} \cdots Y_{n} X_{l-1} \cdots X_{1} \\
& -(-1)^{m+n-1} X_{m} \cdots X_{l+1} Y_{n} \cdots Y_{1} X_{l-1} \cdots X_{1} .
\end{aligned}
$$

However,

$$
\begin{aligned}
& X_{1} \cdots X_{l-1} Y_{1} \cdots Y_{n} X_{l+1} \cdots X_{m}-(-1)^{m+n-1} X_{m} \cdots X_{l+1} Y_{n} \cdots Y_{1} X_{l-1} \cdots X_{1} \\
= & \llbracket X_{1}, \cdots, X_{l-1}, Y_{1} \cdots Y_{n}, X_{l+1}, \cdots, X_{m} \rrbracket_{m+n-1},
\end{aligned}
$$


and, by the same token, we observe that the two middle terms of the right-hand side of the above equation compose into the $m$-nary product

$$
-(-1)^{n} \llbracket X_{1}, \cdots, X_{l-1}, Y_{n}, \cdots, Y_{1}, X_{l+1}, \cdots, X_{m} \rrbracket_{m+n-1} .
$$

Hence equation (18) follows.

It remains to prove that $\left[[\cdots]_{m}\right.$ is an internal operation on $\mathfrak{g}$, by induction on $m$ : for $m=1$, we have $\llbracket X \rrbracket_{1}=2 X \in \mathfrak{g}$ for any $X \in \mathfrak{g}$. Next, let us assume that for every $k \leqslant m-1$ the assertion is true. We distinguish two cases: even and odd values of $m$. Considering first the case when $m$ is even, and letting $J$ be the same matrix as in equation (5), we have

$$
\begin{aligned}
\llbracket X_{1}, \ldots, X_{m-1}, X_{m} \rrbracket_{m} J & =\left(X_{1} \cdots X_{m-1} X_{m}-X_{m} X_{m-1} \cdots X_{1}\right) J \\
& =(-1)^{m} J\left(X_{1}^{\mathrm{T}} \cdots X_{m}^{\mathrm{T}}-X_{m}^{\mathrm{T}} \cdots X_{1}^{\mathrm{T}}\right) \\
& =J \llbracket X_{1}^{\mathrm{T}}, \cdots, X_{m}^{\mathrm{T}} \rrbracket_{m}=-J \llbracket X_{m}^{\mathrm{T}}, \ldots X_{1}^{\mathrm{T}} \rrbracket_{m} \\
& =-J \llbracket X_{1}, \ldots X_{m} \rrbracket_{m}^{\mathrm{T}},
\end{aligned}
$$

by virtue of the alternating symmetry. Hence

$$
\llbracket X_{1}, \ldots, X_{m-1}, X_{m} \rrbracket_{m} \in \mathfrak{g} .
$$

Finally, when $m$ is odd,

$$
\begin{aligned}
\llbracket X_{1}, \ldots, X_{m} \rrbracket_{m} J & =\left(X_{1} \cdots X_{m}+X_{m} \cdots X_{1}\right) J \\
& =-J\left(X_{1}^{\mathrm{T}} \cdots X_{m}^{\mathrm{T}}+X_{m}^{\mathrm{T}} \cdots X_{1}^{\mathrm{T}}\right) \\
& =-J \llbracket\left[X_{1}, \ldots, X_{m} \rrbracket_{m}^{\mathrm{T}} ;\right.
\end{aligned}
$$

hence $\llbracket X_{1}, \ldots, X_{m-1}, X_{m} \rrbracket_{m} \in \mathfrak{g}$. A similar procedure is applied for complex groups, whereby the transpose operator is replaced by the Hermitian operator. It follows that $\llbracket\left[X_{1}, \ldots, X_{m} \rrbracket_{m} \in \mathfrak{g}\right.$ for all values of $m$. The proof is complete.

The importance of hierarchical algebras is therefore self-evident: for quadratic matrix Lie algebras, elements of the form $\llbracket X_{1}, \ldots, X_{m} \rrbracket_{m}$ are precisely the building blocks of the Cayley transform (9).

It is useful to introduce the following definitions.

Definition 2. If $\mathfrak{g}, \mathfrak{h}$ are two hierarchical algebras, we say that $\pi: \mathfrak{g} \rightarrow \mathfrak{h}$ is a hierarchical algebra homomorphism if

$$
\pi\left(\llbracket X_{1}, \ldots, X_{m} \rrbracket_{m}\right)=\llbracket \pi\left(X_{1}\right), \ldots, \pi\left(X_{m}\right) \rrbracket_{m} \in \mathfrak{h}, \quad \forall X_{1}, \ldots, X_{m} \in \mathfrak{g},
$$

for every $m=1,2, \ldots$

Similarly to the definition of free Lie algebras [17], we can introduce the definition for a free hierarchical algebra.

Definition 3. Let $I$ be a finite or a countable set of indices. We say that the hierarchical algebra $\mathfrak{g}$ is free over $I$ if

1. for every $i \in I$ there corresponds $X_{i} \in \mathfrak{g}$, and $X_{i} \neq X_{j}$ except when $i=j$;

2. for any hierarchical algebra $\mathfrak{h}$ for which there exists a function $i \rightarrow Y_{i} \in \mathfrak{h}$, there exists a unique $\pi: \mathfrak{g} \rightarrow \mathfrak{h}$, a hierarchical algebra homomorphism, such that $\pi\left(X_{i}\right)=Y_{i}$. 
Given a set $\left\{X_{i}\right\}_{i \in I}$, denote by $\mathfrak{g}$ the set obtained by the terms $X_{i}$, and also by all the possible combinations of those by means of each $m$-nary operation. By construction, $\mathfrak{g}$ is a hierarchical algebra, and, for any other hierarchical algebra $\mathfrak{h}$ containing the terms $X_{i}$, it is true that $\mathfrak{g} \subseteq \mathfrak{h}$. Hence $\mathfrak{g}$, which is unique up to an isomorphism, is the smallest hierarchical algebra containing the terms $X_{i}$, and will be called a hierarchical algebra generated by the family $\left\{X_{i}\right\}_{i \in I}$ Note that such $\mathfrak{g}$ is free over $I$.

The homomorphism $\pi$ is a representation of a free hierarchical algebra $\mathfrak{g}$ in $\mathfrak{h}$. In concrete terms, if $\mathfrak{h}$ is a quadratic Lie algebra, for a given set of indices $I$ the $\pi\left(X_{i}\right)$ terms might be associated to some function evaluations of $A(t)$ or to some linear combination of those. The $X_{i}$ can be regarded as letters of an alphabet, and the term $\llbracket X_{i_{1}}, \ldots, X_{i_{m}} \rrbracket_{m}$ is a word of length $m$ generated by $X_{i_{1}}, \ldots, X_{i_{m}}$. In the next section we shall be interested in counting the number of words of given length, as well counting the words of a given weight, whenever a weight is initially associated to each of the generators $X_{i}$.

\section{The dimension and a basis of graded linear subspaces}

\subsection{Graded linear spaces}

Let $\mathcal{X}=\left\{X_{1}, X_{2}, \ldots, X_{s}\right\}$ be a set of generators of a free hierarchical algebra $\mathfrak{g}$ and suppose that we are given a map $w: \mathcal{X} \rightarrow \mathbb{N}$. The natural number $w\left(X_{l}\right)$ is called the grade of $X_{l}$. The definition of a grade is propagated in the free hierarchical algebra in a natural manner: provided that $w\left(Y_{j}\right)=v_{j}, j=1,2, \ldots, m$, we set

$$
w\left(\llbracket Y_{1}, Y_{2}, \ldots, Y_{m} \rrbracket_{m}\right)=\sum_{j=1}^{m} v_{j} .
$$

We assume without loss of generality that $w\left(X_{k}\right) \leqslant w\left(X_{l}\right)$ for $k<l$, and stipulate that $w\left(X_{1}\right)=1$. The free hierarchical algebra splits into a direct sum of linear subspaces,

$$
\mathfrak{g}=\bigoplus_{r \geqslant 1} \mathcal{K}_{r}^{s}(\boldsymbol{w})
$$

where $\boldsymbol{w}=\left[w\left(X_{1}\right), w\left(X_{2}\right), \ldots, w\left(X_{s}\right)\right]$ and $\mathcal{K}_{r}^{s}(\boldsymbol{w})$ is the linear subspace generated by all the elements in $\mathfrak{g}$ of grade equal to $r \geqslant 1$. We pose the following two problems.

1. What is $\operatorname{dim} \mathcal{K}_{r}^{s}(\boldsymbol{w})$ ?

2. How do we construct a basis of $\mathcal{K}_{r}^{s}(\boldsymbol{w})$ ?

The raison d'etre for our interest in the dimension and in a basis of $\mathcal{K}_{r}^{s}(\boldsymbol{w})$ is implicit in the discussion of Section 1. Thus, suppose that the function values $A_{1}, A_{2}, \ldots, A_{v}$ generate a free hierarchical algebra, while the multilinear form in equation (10) has been derived by hierarchical-algebra operations. Letting $w\left(A_{l}\right) \equiv 1$, we deduce that

$$
\mathcal{L}\left(A_{k_{1}}, A_{k_{2}}, \ldots, A_{k_{r}}\right) \in \mathcal{K}_{r}^{v}(\mathbf{1})
$$

for all $1 \leqslant k_{1}, k_{2}, \ldots, k_{r} \leqslant v$. This corresponds to the observation that $A_{l}=\mathcal{O}(1)$, for $1 \leqslant l \leqslant v$, implies that

$$
h^{r} \mathcal{L}\left(A_{k_{1}}, A_{k_{2}}, \ldots, A_{k_{r}}\right)=\mathcal{O}\left(h^{r}\right)
$$

and the quadrature (10) is composed of $\mathcal{O}\left(h^{r}\right)$ terms. Having evaluated a basis for $\mathcal{K}_{r}^{v}(\mathbf{1})$, we can construct all the terms in equation (10) out of basis elements by linear combinations. Therefore, it is the dimension of the basis, rather than the number of different combinations 
in quadrature (10) and of different $r$-dimensional integrals that determines the cost of the calculation!

Considerably more important is the case when $A_{1}, A_{2}, \ldots, A_{v}$ are replaced with the linear combinations $B_{0}, B_{1}, \ldots, B_{v-1}$. We let $w\left(B_{l}\right)=l+1$, consistently with $h B_{l}=$ $\mathcal{O}\left(h^{l+1}\right)$, for $l=0,1, \ldots, v-1$. Let $\boldsymbol{w}^{\star}=[1,2, \ldots, v]$. Since

$$
\mathcal{K}_{r}^{v}\left(\boldsymbol{w}^{\star}\right) \ni h^{r} \mathcal{L}\left(B_{l_{1}}, B_{l_{2}}, \ldots, B_{l_{r}}\right)=\mathcal{O}\left(h^{|l|+r}\right),
$$

where $|\boldsymbol{l}|=\sum l_{i}$, we need to retain in equation (11) only terms of grade less than or equal to $2 v$. Therefore, the cost of all the quadrature formulae needed in the implementation of the Cayley expansion (9) is reflected in the dimension of $\mathcal{K}_{r}^{v}\left(\boldsymbol{w}^{\star}\right)$ for $r \leqslant 2 v$.

We mention in passing that our construction is similar to the concept of graded linear spaces in Lie-algebra theory. The dimension of a grade- $r$ subspace $\mathcal{N}_{r}^{s}(\mathbf{1})$, say, of a Lie algebra with initial grades $w\left(X_{l}\right) \equiv 1, l=1,2, \ldots, s$, is given by the familiar Witt formula

$$
\operatorname{dim} \mathcal{N}_{r}^{s}(\mathbf{1})=\frac{1}{r} \sum_{i \mid r} \mu(i) s^{r / i},
$$

where $\mu$ is the Möbius function, while a basis of $\mathcal{N}_{r}^{s}(\mathbf{1})$ can be obtained by algorithms due to Hall and to Lyndon $[\mathbf{1 7}, \mathbf{1 9}]$. The formula (20) and the Hall basis have been generalized to arbitrary sets of weights by Munthe-Kaas and Owren [17]. In particular, we note for future reference that

$$
\operatorname{dim} \mathcal{N}_{r}^{s}(\boldsymbol{w})=\frac{1}{r} \sum_{i \mid r} \mu(i)\left(\sum_{k=1}^{n} \lambda_{k}^{r / i}\right),
$$

where $\lambda_{1}, \lambda_{2}, \ldots, \lambda_{n}, n=\max w_{k}$, are the zeros of

$$
p(z)=z^{n}-\sum_{i=1}^{s} z^{n-w_{i}}
$$

\subsection{A generating function}

Let $w_{l}=w\left(X_{l}\right)$, for $l=1,2, \ldots, s$. In this subsection we explicitly construct a generating function of the sequence $\left\{d_{r}^{s}\right\}_{r} \geqslant 1$, where

$$
d_{r}^{s}=\operatorname{dim} \mathcal{K}_{r}^{v}(\boldsymbol{w})
$$

To this end, however, we first require a technical result relating to the form of the elements in the free hierarchical algebra $\mathfrak{g}$.

Definition 4. We say that $Y \in \mathfrak{g}$, where $\mathfrak{g}$ is a free hierarchical algebra generated by $X_{1}, X_{2}, \ldots, X_{s}$, is primitive if there exist $m \geqslant 1$ and $i_{1}, i_{2}, \ldots, i_{m} \in\{1,2, \ldots, s\}$ such that $Y=\llbracket X_{i_{1}}, X_{i_{2}}, \ldots, X_{i_{s}} \rrbracket_{m}$. Moreover, we say that a basis $\mathcal{B}_{r}^{s}$ of $\mathcal{K}_{r}^{s}(\boldsymbol{w})$ is primitive if all its elements are primitive.

As an example of a non-primitive element, consider

$$
\llbracket X_{i_{1}}, X_{i_{2}}, \llbracket\left[X_{i_{3}}, X_{i_{4}} \rrbracket_{2} \rrbracket_{3}\right.
$$

Lemma 3. Every $Y \in \mathfrak{g}$ is a linear combination of primitive elements. 
Proof. The lemma is proved by induction on the grade. Clearly, $\llbracket X_{1} \rrbracket_{1}$ is a primitive element. Suppose thus that the lemma is true for all elements of grade less than or equal to $n-1$, where $n \geqslant 2$, and suppose that $Y \in \mathcal{K}_{n}^{s}(\boldsymbol{w})$ and is non-primitive. Since $\mathfrak{g}$ is the algebra generated by $X_{1}, \ldots, X_{s}$, there exist indices $k_{1}, \ldots k_{m}$, for $m<n$, such that

$$
Y=\llbracket \llbracket X_{i_{k_{1}, 1}}, \ldots, X_{i_{k_{1}, k_{1}}} \rrbracket_{k_{1}}, \llbracket X_{i_{k_{2}, 1}}, \ldots, X_{i_{k_{2}, k_{2}}} \rrbracket_{k_{2}}, \ldots, \llbracket X_{i_{k_{m}, 1}}, \ldots, X_{i_{k_{m}, k_{m}}} \rrbracket_{k_{m}} \rrbracket_{m},
$$

where $\llbracket X_{i_{k_{j}, 1}}, \ldots, X_{i_{k_{j}, k_{j}}} \rrbracket_{k_{j}}$ has grade $v_{j}<n$ for all $1 \leqslant j \leqslant m$ and $v_{1}+\cdots+v_{m}=n$. Note that [[ $X_{i_{k_{j}, 1}}, \ldots, X_{i_{k_{j}}, k_{j}} \rrbracket_{k_{j}}$ is a primitive element for all $j \leqslant m$. The lemma follows by the multilinearity (17) and the hierarchy condition (18).

Definition 5. Given $Y \in \mathfrak{g}$ we say that $Y$ is of length $m$ if it is primitive and $Y=$ $\llbracket X_{i_{1}}, \ldots, X_{i_{m}} \rrbracket_{m}$. If $Y$ is non-primitive, its length equals the maximum of the lengths of the primitive words that compose it.

As an example, the non-primitive word $\llbracket X_{i_{1}}, X_{i_{2}}, \llbracket X_{i_{3}}, X_{i_{4}} \rrbracket_{2} \rrbracket_{3}$ has length four, since it can be decomposed into

$$
\llbracket X_{i_{1}}, X_{i_{2}}, X_{i_{3}}, X_{i_{4}} \rrbracket_{4}-\llbracket\left[X_{i_{1}}, X_{i_{2}}, X_{i_{4}}, X_{i_{3}} \rrbracket_{4}\right.
$$

a linear combination of primitive words both having length equal to four.

It is a consequence of Lemma 3 that there exists a primitive basis $\mathcal{B}_{r}^{s}$ of $\mathcal{K}_{r}^{s}(\boldsymbol{w})$. For every $m \geqslant 1$ we denote by $v_{r, m}^{[s]} \geqslant 0$ the number of $m$-nary terms in $\mathcal{B}_{r}^{s}$; that is, of terms of the form $\left[\left[X_{i_{1}}, X_{i_{2}}, \ldots, X_{i_{m}} \rrbracket_{m}\right.\right.$. Clearly,

$$
d_{r}^{s}=\sum_{m=1}^{\infty} v_{r, m}^{[s]} .
$$

Hence, defining the generating functions

$$
\Theta_{m}(z):=\sum_{r=1}^{\infty} v_{r, m}^{[s]} z^{r}, \quad m \geqslant 1, \quad W(z)=\sum_{r=1}^{\infty} d_{r}^{s} z^{r},
$$

we obtain

$$
W(z)=\sum_{m=1}^{\infty} \Theta_{m}(z) .
$$

Our intention is to obtain each function $\Theta_{m}$, for $m \geqslant 1$, in a closed form. To this end we commence by observing that

$$
\Theta_{1}(z)=q(z):=\sum_{i=1}^{s} z^{w_{i}}
$$

The reason is that $\Theta_{1}$ contains precisely the contribution of all unary terms, and hence of the generators themselves, and each $X_{i}$ adds $z^{w_{i}}$ to the sum.

We next obtain $\Theta_{m}$ s recursively, distinguishing between even and odd indices $m$.

Case 1: $m=2 M$.

Each term $\llbracket X_{i_{1}}, X_{i_{2}}, \ldots, X_{i_{2 M}} \rrbracket_{2 M}$ in a primitive basis contributes an expression of the form $z^{w_{i_{1}}+w_{i_{2}}+\cdots+w_{i_{2 M}}}$ to $\Theta_{2 M}$. We need to single out the indices $i_{1}, i_{2}, \ldots, i_{2 M} \in\{1,2, \ldots, s\}$ that are allowed in the basis; that is, those that do not interfere with linear independence. 


\section{Graded Lie algebras}

Choose $t \in\{0,1, \ldots, M-1\}$ and assume that

$$
i_{1}=i_{2 M}, \quad i_{2}=i_{2 M-1}, \quad \ldots, \quad i_{t}=i_{2 M+1-t}, \quad i_{t+1}<i_{2 M-t} .
$$

In that case any choice of $1 \leqslant i_{t+2}, \ldots, i_{2 M-t-1} \leqslant s$ leads to a linearly independent term. Therefore the contribution to $\Theta_{2 M}$ is

$$
\begin{aligned}
& \sum_{i_{1}=1}^{s} \cdots \sum_{i_{t}=1}^{s} \sum_{i_{t+1}=1}^{s-1} \sum_{i_{2 M-t}=i_{t+1}+1}^{s} \sum_{i_{t+2}=1}^{s} \cdots \sum_{i_{2 M-t-1}=1}^{s} z^{2\left(w_{i_{1}}+\cdots+w_{i_{t}}\right)+w_{t+1}+\cdots+w_{2 M-t}} \\
= & \left(\sum_{i=1}^{s} z^{2 w_{i}}\right)^{t}\left(\sum_{i=1}^{s} z^{y_{i}}\right)^{2 M-2 t-2} \sum_{k=1}^{s-1} \sum_{l=k+1}^{s} z^{w_{k}+w_{l}} \\
= & {\left[q\left(z^{2}\right)\right]^{t}[q(z)]^{2 M-2 t-2} \sum_{k=1}^{s-1} \sum_{l=k+1}^{s} z^{w_{k}+w_{l}} . }
\end{aligned}
$$

Moreover,

$$
\sum_{k=1}^{s-1} \sum_{l=k+1}^{s} z^{w_{k}+w_{l}}=\sum_{k=2}^{s} \sum_{l=1}^{k-1} z^{w_{k}+w_{l}}
$$

therefore

$$
\begin{aligned}
\sum_{k=1}^{s-1} \sum_{l=k+1}^{s} z^{w_{k}+w_{l}} & =\frac{1}{2}\left(\sum_{k=1}^{s-1} \sum_{l=k+1}^{s} z^{w_{k}+w_{l}}+\sum_{k=2}^{s} \sum_{l=1}^{k-1} z^{w_{k}+w_{l}}\right) \\
& =\frac{1}{2}\left(\sum_{k=1}^{s} \sum_{l=1}^{s} z^{w_{k}+w_{l}}-\sum_{k=1}^{s} z^{2 w_{k}}\right)=\frac{1}{2}\left\{[q(z)]^{2}-q\left(z^{2}\right)\right\} .
\end{aligned}
$$

We thus deduce that the contributions of all the terms for this value of $t$ is

$$
\begin{aligned}
& \frac{1}{2}\left[q\left(z^{2}\right)\right]^{t}[q(z)]^{2 M-2 t-2}\left\{[q(z)]^{2}-q\left(z^{2}\right)\right\} \\
= & \frac{1}{2}\left\{\left[q\left(z^{2}\right)\right]^{t}[q(z)]^{2 M-2 t}-\left[q\left(z^{2}\right)\right]^{t+1}[q(z)]^{2 M-2 t-2}\right\} .
\end{aligned}
$$

All possible candidates for elements in a primitive basis are obtained by allowing $t$ in assumption (24) to range across $\{0,1, \ldots, M-1\}$. Note that we can always assume that $i_{t+1}<i_{2 M-t}$; otherwise we use alternating symmetry (16) to obtain a linearly dependent term. Therefore $\Theta_{2 M}$ can be obtained by summing up in $t$, series telescope and

$$
\begin{aligned}
\Theta_{2 M}(z) & =\frac{1}{2} \sum_{t=0}^{M-1}\left\{\left[q\left(z^{2}\right)\right]^{t}[q(z)]^{2 M-2 t}-\left[q\left(z^{2}\right)\right]^{t+1}[q(z)]^{2 M-2 t-2}\right\} \\
& =\frac{1}{2}\left\{[q(z)]^{2 M}-\left[q\left(z^{2}\right)\right]^{M}\right\} .
\end{aligned}
$$

Case 2: $m=2 M+1$.

This case is very similar, except for one important detail. We assemble all linearly independent terms in $\llbracket X_{i_{1}}, X_{i_{2}}, \ldots, X_{i_{2 M+1}} \rrbracket_{2 M+1}$. For every $t=0,1, \ldots, M-1$ we take, similarly to assumption (24),

$$
i_{1}=i_{2 M+1}, \quad i_{2}=i_{2 M}, \quad \ldots, \quad i_{t}=i_{2 M-t+2}, \quad i_{t+1}<i_{2 M-t+1},
$$




\section{Graded Lie algebras}

whence the contribution to $\Theta_{2 M+1}$ is

$$
\frac{1}{2}\left\{\left[q\left(z^{2}\right)\right]^{t}[q(z)]^{2 M-2 t+1}-\left[q\left(z^{2}\right)\right]^{t+1}[q(z)]^{2 M-2 t-1}\right\} ;
$$

the algebra is virtually identical to the previous case. However, if

$$
i_{1}=i_{2 M+1}, \quad i_{2}=i_{2 M}, \quad \ldots, \quad i_{M}=i_{M+2}
$$

then the middle index $i_{M+1}$ can be allowed to range across all of $\{1,2, \ldots, s\}$, and this contributes

$$
\left[q\left(z^{2}\right)\right]^{M} q(z)
$$

to $\Theta_{2 M+1}$. Summing up in $t$ leads at once to

$$
\Theta_{2 M+1}(z)=\frac{1}{2}\left\{[q(z)]^{2 M+1}+q(z)\left[q\left(z^{2}\right)\right]^{M}\right\} .
$$

Note that this is consistent with equation (23), and therefore valid for all $M \geqslant 0$.

We can now substitute equations (25) and (26) in equation (22), and the outcome is the generating function

$$
\begin{aligned}
W(z) & =\frac{1}{2} \sum_{m=1}^{\infty}[q(z)]^{m}+\frac{1}{2} q(z) \sum_{M=0}^{\infty}\left[q\left(z^{2}\right)\right]^{M}-\frac{1}{2} \sum_{M=1}^{\infty}\left[q\left(z^{2}\right)\right]^{M} \\
& =\frac{1}{2} \frac{q(z)}{1-q(z)}+\frac{1}{2} \frac{q(z)}{1-q\left(z^{2}\right)}-\frac{1}{2} \frac{q\left(z^{2}\right)}{1-q\left(z^{2}\right)} \\
& =\frac{q(z)-\frac{1}{2} q\left(z^{2}\right)-\frac{1}{2}[q(z)]^{2}}{[1-q(z)]\left[1-q\left(z^{2}\right)\right]} .
\end{aligned}
$$

\subsection{The dimension of graded subspaces}

We intend in this subsection to find explicitly $d_{r}^{s}=\operatorname{dim} \mathcal{K}_{r}^{s}$. The point of departure is the explicit formula (27) for the generating function. To expand $W(z)$ in a Taylor series, we need to invert polynomials $1-q(z)$ and $1-q\left(z^{2}\right)$. This issue is explored in the next lemma.

Lemma 4. Given any distinct $\theta_{1}, \theta_{2}, \ldots, \theta_{n} \in \mathbb{C} \backslash\{0\}$, it is true that

$$
\prod_{l=1}^{n}\left(1-\theta_{l} z\right)^{-1}=\sum_{k=0}^{\infty}\left[\sum_{l=1}^{n} \frac{\theta_{l}^{k+n-1}}{\omega_{n}^{\prime}\left(\theta_{l}\right)}\right] z^{k}, \quad z \in \mathbb{C},
$$

where $\omega_{n}(z)=\prod_{k=1}^{n}\left(z-\theta_{k}\right)$.

Proof. The expansion (28) is a straightforward consequence of the residuum theorem. The poles of $f(z)=\prod_{l=1}^{n}\left(1-\theta_{l} z\right)^{-1}$ being simple, it is true that

$$
\prod_{l=1}^{n}\left(1-\theta_{l} z\right)^{-1}=\sum_{l=1}^{n} \frac{f_{l}}{1-\theta_{l} z}
$$

where

$$
f_{l}=\lim _{z \rightarrow \theta_{l}^{-1}}\left(1-\theta_{l} z\right) f(z)=\frac{\theta_{l}^{n-1}}{\omega_{n}^{\prime}\left(\theta_{l}\right)} .
$$

The lemma follows by expanding each $1-\theta_{l} z$ as a geometric series. 


\section{Graded Lie algebras}

Letting $n=w_{s}=\max w_{k}$ be the degree of the polynomial $q$, we use expansion (28) with $\varphi_{n}(z)=[1-q(z)]^{-1}$. Therefore

$$
\omega_{n}(z)=z^{n}\left[1-q\left(z^{-1}\right)\right]=z^{n}-\sum_{k=1}^{s} z^{n-w_{k}}=p(z),
$$

the polynomial $p$ having already been encountered in equation (21). We again denote its zeros by $\lambda_{1}, \lambda_{2}, \ldots, \lambda_{n}$, whereby, by equation (28),

$$
\frac{1}{1-q(z)}=\sum_{k=0}^{\infty}\left[\sum_{l=1}^{n} \frac{\lambda_{l}^{k+1}}{q^{\prime}\left(\lambda_{l}^{-1}\right)}\right] z^{k} \text {. }
$$

The expansion of $\left[1-q\left(z^{2}\right)\right]^{-1}$ can be similarly obtained, replacing $z$ with $z^{2}$.

Theorem 5. Suppose that all the zeros $\lambda_{1}, \lambda_{2}, \ldots, \lambda_{n}$ of p away from the origin are distinct. The dimensions of the graded subspaces are

$$
\begin{aligned}
d_{2 R}^{S} & =\frac{1}{2} \sum_{l=1}^{n} \frac{1}{q^{\prime}\left(\lambda_{l}^{-1}\right)}\left\{\lambda_{l}^{2 R+1}-\lambda_{l}^{R+1}+\frac{1}{2} \lambda_{l}^{R+1}\left[q\left(\lambda_{l}^{-1 / 2}\right)+q\left(-\lambda_{l}^{-1 / 2}\right)\right]\right\}, \\
d_{2 R+1}^{S} & =\frac{1}{2} \sum_{l=1}^{n} \frac{1}{q^{\prime}\left(\lambda_{l}^{-1}\right)}\left\{\lambda_{l}^{2 R+2}+\frac{1}{2} \lambda_{l}^{R+3 / 2}\left[q\left(\lambda_{l}^{-1 / 2}\right)-q\left(-\lambda_{l}^{-1 / 2}\right)\right]\right\} .
\end{aligned}
$$

Proof. Bearing in mind that $q(z)=1-\prod_{l=1}^{n}\left(1-\lambda_{l} z\right)$, we replace function (27) with

$$
W(z)=\frac{1}{2}\left[\frac{1}{1-q(z)}-\frac{1-q(z)}{1-q\left(z^{2}\right)}\right]=\frac{1}{2}\left[\frac{1}{1-q(z)}-\frac{\prod_{l=1}^{n}\left(1-\lambda_{l} z\right)}{1-q\left(z^{2}\right)}\right] .
$$

Let

$$
\prod_{l=1}^{n}\left(1-\lambda_{l} z\right)=\sum_{j=0}^{n} u_{j} z^{j}
$$

Then

$$
\begin{aligned}
\frac{\prod_{l=1}^{n}\left(1-\lambda_{l} z\right)}{1-q\left(z^{2}\right)} & =\sum_{k=0}^{\infty} \varphi_{n, k} \sum_{j=0}^{n} u_{j} z^{2 k+j}=\sum_{j=0}^{\infty}\left(\sum_{k=\lfloor(j-n+1) / 2\rfloor}^{\lfloor j / 2\rfloor} u_{j-2 k} \varphi_{n, k}\right) z^{j} \\
& =\sum_{j=0}^{\infty}\left[\sum_{l=1}^{n} \frac{1}{q^{\prime}\left(\lambda_{l}^{-1}\right)} \sum_{k=\lfloor(j-n+1) / 2\rfloor}^{\lfloor j / 2\rfloor} u_{j-2 k} \lambda_{l}^{k+1}\right] z^{j},
\end{aligned}
$$

where we set $u_{j}=0$ outside the range $0 \leqslant j \leqslant n$.

We next consider the sum

$$
g_{n, j}(\xi):=\sum_{k=\lfloor(j-n+1) / 2\rfloor}^{\lfloor j / 2\rfloor} u_{j-2 k} \xi^{k+1}, \quad n, j \geqslant 0, \quad \xi \in \mathbb{C} .
$$

By straightforward computation

$$
g_{2 N, 2 J}(\xi)=\sum_{k=J-N}^{J} u_{2 J-2 k} \xi^{k+1}=\xi^{J+1} \sum_{k=0}^{N} u_{2 k} \xi^{-k}
$$




$$
\begin{aligned}
g_{2 N, 2 J+1}(\xi) & =\sum_{k=J-N+1}^{J} u_{2 J+1-2 k} \xi^{k+1}=z^{J+1} \sum_{k=0}^{N-1} u_{2 k+1} \xi^{-k}, \\
g_{2 N+1,2 J}(\xi) & =\sum_{k=J-N}^{J} u_{2 J-2 k} \xi^{k+1}=\xi^{J+1} \sum_{k=0}^{N} u_{2 k} \xi^{-k}, \\
g_{2 N+1,2 J+1}(\xi) & =\sum_{k=J-N}^{J} u_{2 J-2 k+1} \xi^{k+1}=\xi^{J+1} \sum_{k=0}^{N} u_{2 k+1} \xi^{-k} .
\end{aligned}
$$

We thus deduce from equation (32) that

$$
\begin{gathered}
g_{n, 2 J}(\xi)=\frac{1}{2} \xi^{J+1}\left[\prod_{i=1}^{n}\left(1-\frac{\lambda_{i}}{\xi^{1 / 2}}\right)+\prod_{i=1}^{n}\left(1+\frac{\lambda_{i}}{\xi^{1 / 2}}\right)\right], \\
g_{n, 2 J+1}(z)=\frac{1}{2} z^{J+3 / 2}\left[\prod_{i=1}^{n}\left(1-\frac{\lambda_{i}}{\xi^{1 / 2}}\right)-\prod_{i=1}^{n}\left(1+\frac{\lambda_{i}}{\xi^{1 / 2}}\right)\right]
\end{gathered}
$$

and, if we let $\xi=\lambda_{l}$ and $\prod_{j=1}^{n}\left(1-\lambda_{j} z\right)=1-q(z)$, the explicit formulae (29) and (30) follow from substitution in equation (31).

\subsection{Examples of graded subspaces}

The simplest special case is when the grades of the generators are equal, $w_{l} \equiv 1$, $l=1,2, \ldots, s$. In that case $q(z)=s z$; therefore $p(z)=z^{n}-s z^{n-1}$, and we have $n=1$, $\lambda_{1}=s$. Substitution in formulae (29) and (30) affirms that

$$
d_{2 R-1}^{S}=\frac{1}{2}\left(s^{2 R-1}+s^{R}\right), \quad d_{2 R}^{S}=\frac{1}{2}\left(s^{2 R}-s^{R}\right), \quad R \geqslant 1 .
$$

Table 1 displays the dimensions of subspaces of graded Lie and hierarchical algebras with $s=2$ and $s=3$. Note that equations (20) and (33) imply that

$$
\operatorname{dim} \mathcal{N}_{r}^{s}(\mathbf{1}) \approx \frac{s^{r}}{r}, \quad \operatorname{dim} \mathcal{K}_{r}^{s}(\mathbf{1}) \approx \frac{s^{r}}{2}, \quad r \gg 1,
$$

consistently with Table 1 .

Table 1: The dimensions of graded subspaces of Lie and hierarchical algebras: $w_{l} \equiv 1$.

\begin{tabular}{c|rrrrrrrrrr}
\hline Subspaces & \multicolumn{10}{|c}{$r$} \\
\cline { 2 - 11 } & 1 & 2 & 3 & 4 & 5 & 6 & 7 & 8 & 9 & 10 \\
\hline $\operatorname{dim} \mathcal{N}_{r}^{2}$ & 2 & 1 & 2 & 3 & 6 & 9 & 18 & 30 & 56 & 99 \\
$\operatorname{dim} \mathcal{K}_{r}^{2}$ & 2 & 1 & 6 & 6 & 20 & 28 & 72 & 120 & 272 & 496 \\
$\operatorname{dim} \mathcal{N}_{r}^{3}$ & 3 & 3 & 8 & 18 & 48 & 116 & 312 & 810 & 2184 & 5880 \\
$\operatorname{dim} \mathcal{K}_{r}^{3}$ & 3 & 3 & 18 & 36 & 135 & 351 & 1134 & 3240 & 9963 & 29403 \\
\hline
\end{tabular}

In our second example we let $s=2$ and consider $w_{1}=1, w_{2}=n \geqslant 2$. Therefore $q(z)=z+z^{n}$, an $n$ th-degree polynomial, and the zeros $\lambda_{1}, \lambda_{2}, \ldots, \lambda_{n}$ obey $\lambda_{l}^{n}-\lambda_{l}^{n-1}=1$. It is easy to verify that $q^{\prime}\left(\lambda_{l}^{-1}\right)=1-n+n \lambda_{l}$, that all the zeros are simple, and that $\frac{1}{2}[q(z)+q(-z)]=\left\{\begin{array}{ll}z^{n}, & n \text { even, } \\ 0, & n \text { odd; }\end{array} \quad \frac{1}{2}[q(z)-q(-z)]= \begin{cases}z, & n \text { even }, \\ z+z^{n}, & n \text { odd } .\end{cases}\right.$ 


\section{Graded Lie algebras}

We deduce that for even $n$,

$$
\begin{aligned}
\operatorname{dim} \mathcal{K}_{2 R}^{2} & =\frac{1}{2} \sum_{l=1}^{n} \frac{1}{1-n+n \lambda_{l}}\left(\lambda_{l}^{2 R+1}-\lambda_{l}^{R+1}+\lambda_{l}^{R+1-n / 2}\right) \\
\operatorname{dim} \mathcal{K}_{2 R+1}^{2} & =\frac{1}{2} \sum_{l=1}^{n} \frac{1}{1-n+n \lambda_{l}}\left(\lambda_{l}^{2 R+2}+\lambda_{l}^{R+1}\right),
\end{aligned}
$$

while odd $n$ yields

$$
\begin{aligned}
\operatorname{dim} \mathcal{K}_{2 R}^{2} & =\frac{1}{2} \sum_{l=1}^{n} \frac{1}{1-n+n \lambda_{l}}\left(\lambda_{l}^{2 R+1}-\lambda_{l}^{R+1}\right), \\
\operatorname{dim} \mathcal{K}_{2 R+1}^{2} & =\frac{1}{2} \sum_{l=1}^{n} \frac{1}{1-n+n \lambda_{l}}\left[\lambda_{l}^{2 R+2}+\lambda_{l}^{R+1}+\lambda_{l}^{R+1-(n-1) / 2}\right] .
\end{aligned}
$$

It follows from the above formula and from equation (21) that

$$
\operatorname{dim} \mathcal{N}_{r}^{2}([1, n]) \approx \frac{\lambda_{\max }^{r}}{r}, \quad \operatorname{dim} \mathcal{K}_{r}^{2}([1, n]) \approx \frac{\lambda_{\max }^{r+1}}{1-n+n \lambda_{\max }}, \quad r \gg 1,
$$

where $\lambda_{\max }=\max _{l=1,2, \ldots, n}\left|\lambda_{l}\right|$.

Our third and last example is probably the most important within the context of geometric integration (cf. the discussion in Subsection 3.1): $w_{l}=l, l=1,2, \ldots, s$. Therefore

$$
q(z)=\sum_{l=1}^{s} z^{l}=\frac{z-z^{s+1}}{1-z}
$$

and $n=s$. Moreover, if $q\left(\lambda_{l}^{-1}\right)=1$ then $\lambda_{l}^{-s-1}=2 \lambda_{l}-1$; therefore

$$
q^{\prime}\left(\lambda_{l}^{-1}\right)=\frac{1-(s+1) \lambda_{l}^{-s}+s \lambda_{l}^{-s-1}}{\left(1-\lambda_{l}^{-1}\right)^{2}}=\lambda_{l} \frac{2 s-(1+s) \lambda_{l}}{1-\lambda_{l}} .
$$

Thus, $q^{\prime}\left(\lambda_{l}^{-1}\right)=0$ (hence, a multiple root) may occur only for $\lambda_{l}=[(s+1) / 2]^{1 / s}>0$. This is impossible according to Lemma 6 below, and we deduce that all the $\lambda_{l}$ are distinct. Letting $s$ be even, we have

$$
\frac{1}{2}[q(z)+q(-z)]=\frac{z^{2}-z^{s+2}}{1-z^{2}}, \quad \frac{1}{2}[q(z)-q(-z)]=\frac{z-z^{s+1}}{1-z^{2}} ;
$$

therefore, again letting $\boldsymbol{w}^{\star}=[1,2, \ldots, s]$,

$$
\begin{aligned}
\operatorname{dim} \mathcal{K}_{2 R}^{s}\left(\boldsymbol{w}^{\star}\right) & =\frac{1}{2} \sum_{l=1}^{s} \frac{1-\lambda_{l}}{2 s-(1+s) \lambda_{l}}\left(\lambda_{l}^{2 R}-\lambda_{l}^{R}+\lambda_{l}^{R} \frac{\lambda_{l}^{-s / 2}-1}{1-\lambda_{l}}\right) \\
\operatorname{dim} \mathcal{K}_{2 R+1}^{s}\left(\boldsymbol{w}^{\star}\right) & =\frac{1}{2} \sum_{l=1}^{s} \frac{1-\lambda_{l}}{2 s-(1+s) \lambda_{l}}\left(\lambda_{l}^{2 R+1}+\lambda_{l}^{R+1} \frac{\lambda_{l}^{-s / 2}-1}{1-\lambda_{l}}\right) .
\end{aligned}
$$

Likewise, for odd $s$,

$$
\frac{1}{2}[q(z)+q(-z)]=\frac{z^{2}-z^{s+1}}{1-z^{2}}, \quad \frac{1}{2}[q(z)-q(-z)]=\frac{z-z^{s+2}}{1-z^{2}},
$$




\section{Graded Lie algebras}

and we obtain

$$
\begin{aligned}
\operatorname{dim} \mathcal{K}_{2 R}^{s}\left(\boldsymbol{w}^{\star}\right) & =\frac{1}{2} \sum_{l=1}^{s} \frac{1-\lambda_{l}}{2 s-(1+s) \lambda_{l}}\left(\lambda_{l}^{2 R}-\lambda_{l}^{R}+\lambda_{l}^{R} \frac{\lambda_{l}^{-(s-1) / 2}-1}{1-\lambda_{l}}\right), \\
\operatorname{dim} \mathcal{K}_{2 R+1}^{s}\left(\boldsymbol{w}^{\star}\right) & =\frac{1}{2} \sum_{l=1}^{s} \frac{1-\lambda_{l}}{2 s-(1+s) \lambda_{l}}\left(\lambda_{l}^{2 R}+\lambda_{l}^{R} \frac{\lambda_{l}^{-(s-3) / 2}-1}{1-\lambda_{l}}\right) .
\end{aligned}
$$

We have already observed in equations (34) and (35) that the asymptotic behaviour of the sequences $\left\{\mathcal{N}_{r}^{s}\right\}_{r} \geqslant 1$ and $\left\{\mathcal{K}_{r}^{s}\right\}_{r} \geqslant 1$ for large $r$ is predictable, and that the former increases somewhat more slowly. In the present case, letting $\lambda_{\max }$ be the largest zero, we have

$$
\operatorname{dim} \mathcal{N}_{r}^{s}\left(\boldsymbol{w}^{\star}\right) \approx \frac{1}{r} \lambda_{\text {max }}^{r}, \quad \operatorname{dim} \mathcal{K}_{r}^{s}\left(\boldsymbol{w}^{\star}\right) \approx \frac{1}{2} \frac{\lambda_{\max }-1}{(1+s) \lambda_{\max }-2 s} \lambda_{\max }^{r}, \quad r \gg 1 .
$$

To estimate $\lambda_{\max }$ we note that $\lim _{s \rightarrow \infty} q(z)=z(1-z)^{-1}$. Therefore all the $\lambda_{l}$ except for one tend to zero, while, without loss of generality, $\lambda_{\max }=\lambda_{1} \rightarrow 2$. More precisely, $\lambda_{1}^{s+1}-2 \lambda_{1}^{s}+1=0$ implies that

$$
\lambda_{1}=2-\frac{1}{s 2^{s}}[1+o(1)], \quad s \gg 1 .
$$

Therefore, approximately,

$$
\operatorname{dim} \mathcal{N}_{r}^{s}\left(\boldsymbol{w}^{\star}\right) \approx \frac{2^{r}}{r}, \quad \operatorname{dim} \mathcal{K}_{r}^{s}\left(\boldsymbol{w}^{\star}\right) \approx 2^{r-2}, \quad r, s \gg 1
$$

\subsection{Asymptotic behaviour}

We have already observed in equations (34), (35) and (36) that the dimension of a graded subspace of a Lie algebra is consistently smaller for large $r$ than its hierarchical-algebra counterpart.

\section{Lemma 6. Let $s \geqslant 2$. The equation}

$$
q(z)=1, \quad \text { where } \quad q(z)=\sum_{l=1}^{s} z^{w_{l}},
$$

possesses a unique positive root $\eta \in(0,1)$. This root is simple and all other roots of equation (37) reside in the open disc $|z|>\eta$.

Proof. Since $q(0)=0$ and $q(1)=s \geqslant 2$, it follows that $q(z)-1$ changes sign in $(0,1)$. Moreover, $q^{\prime}(z)>0$ for $z \geqslant 0$; therefore this root, which we denote by $\eta$, is simple, and it is the unique positive root of equation (37).

Let $n=\max w_{l}$. If $n=1$ then $q(z)=s z$ for $\eta=1 / s$, and there are no other roots. The lemma is certainly true in this case; hence we may assume in the remainder of the proof that $n \geqslant 2$. Let $\xi=|\xi| \mathrm{e}^{\mathrm{i} \theta}$ be another root of equation (37), and note that we have already proved that $\theta \in(0,2 \pi)$. Since $q(z)$ is a linear combination of at least two different powers of $z$, it is true that

$$
|q(\xi)|=\left.\left.\left|\sum_{l=1}^{s}\right| \xi\right|^{w_{l}} \mathrm{e}^{\mathrm{i} \theta_{l}}\left|<\sum_{l=1}^{s}\right| \xi\right|^{w_{l}}=q(|\xi|) .
$$

Since $\xi$ is a root of equation (37), we deduce that $q(|\xi|)>1$. Because of the monotonicity of $q$ in $[0, \infty)$, we conclude that $|\xi|>\eta$, and the lemma follows. 
Theorem 7. Suppose that the roots $\lambda_{1}, \lambda_{2}, \ldots, \lambda_{n}$ are distinct. For $r \gg 1$ it is true that

$$
\frac{\operatorname{dim} \mathcal{K}_{r}^{s}(\boldsymbol{w})}{\operatorname{dim} \mathcal{N}_{r}^{s}(\boldsymbol{w})}=\frac{r}{2 \eta q(\eta)}[1+o(1)]
$$

where $\eta$ is the positive root of equation (37).

Proof. Since $1 / \eta$ dominates the other roots $\lambda_{l}$, we deduce from equations (21), (29) and (30) that, for $r \gg 1$,

$$
\begin{aligned}
\operatorname{dim} \mathcal{N}_{r}^{s}(\boldsymbol{w}) & \approx \frac{\mu(1) \eta^{-r}}{r}=\frac{1}{r \eta^{r}} \\
\operatorname{dim} \mathcal{K}_{r}^{s}(\boldsymbol{w}) & \approx \frac{\eta^{-r-1}}{2 q^{\prime}(\eta)}=\frac{1}{2 q^{\prime}(\eta) \eta^{r+1}} .
\end{aligned}
$$

The estimate (38) follows easily.

\subsection{Multiple roots}

Much of the analysis of Subsections 3.3 and 3.5 is based on the assumption that all the roots of the equation $q(z)=1$ are simple. It is easy to construct as example of a graded hierarchical algebra with multiple roots by taking $s=9$ and

$$
w\left(X_{1}\right)=1, \quad w\left(X_{2}\right)=\cdots=w\left(X_{6}\right)=2, \quad w\left(X_{7}\right)=w\left(X_{8}\right)=w\left(X_{9}\right)=3 .
$$

Therefore $q(z)=z+5 z^{2}+3 z^{3}$ and

$$
q(z)-1=\left((z+1)^{2}(3 z-1),\right.
$$

with a double root at $z=-1$. The generating function (27), of course, remains valid and we have

$$
\begin{aligned}
W(z)= & \frac{z+4 z^{2}-2 z^{3}-18 z^{4}-15 z^{5}-6 z^{6}}{(1+z)^{2}(1-3 z)(1+z)^{2}\left(1-3 z^{2}\right)} \\
= & \frac{1}{8} \frac{1}{(1+z)^{2}}+\frac{3}{32} \frac{1}{1+z}+\frac{9}{32} \frac{1}{1-3 z}+\frac{3}{16} \frac{1+3 z}{1-3 z^{2}}-\frac{1}{16} \frac{5-3 z}{1+z^{2}} \\
& -\frac{1}{8} \frac{3+2 z-3 z^{2}}{\left(1+z^{2}\right)^{2}} \\
= & z+5 z^{2}+9 z^{3}+23 z^{4}+72 z^{5}+214 z^{6}+630 z^{7}+1858 z^{8}+5579 z^{9}+\cdots .
\end{aligned}
$$

This particular example is of little independent interest except that it indicates how to generalise our analysis to the case of multiple roots. The explicit expressions (29) and (30) are no longer valid, but they can be generalised with moderate effort. Moreover, note that Lemma 6 remains valid, and it is easy to confirm that the asymptotic estimate (38) is true also for multiple roots.

\subsection{A basis of $\mathcal{K}_{r}^{s}(\boldsymbol{w})$}

Our method for the formation of a basis of the graded linear subspace $\mathcal{K}_{r}^{s}(\boldsymbol{w})$ is nothing but an algorithmic rendering of the argument in Subsection 3.2 which has led to the generating function (27). In essence, we choose a basis of primitive elements, removing all elements that are linearly dependent by virtue of the alternating symmetry (16). Note that this is, in a manner of speaking, the exact opposite of the standard procedure for the 


\section{Graded Lie algebras}

formation of Hall or Lyndon bases of graded subspaces in Lie algebras. The basic building blocks in the latter are iterated commutators of generators, terms of the form

$$
\left[X_{i_{1}},\left[X_{i_{2}},\left[\ldots,\left[X_{i_{m-1}}, X_{i_{m}}\right] \cdots\right]\right]\right],
$$

where $i_{1}, i_{2}, \ldots, i_{m} \in\{1,2, \ldots, s\}$ and linear dependencies occur by virtue of skewsymmetry of the commutator and of the Jacobi identity [17].

Henceforth, we denote our basis of $\mathcal{K}_{r}^{s}(\boldsymbol{w})$ by $\mathcal{B}_{r}^{s}$.

\section{Algorithm 1. Looping through r}

Step 1. We initially let $\mathcal{B}_{r, 1}^{s}$ consist of all the generators $X_{l}$ such that $w_{l}=r$. If there are no such generators, we let $\mathcal{B}_{r, 1}^{s}=\varnothing$.

Step 2. For every even $m=2 M \leqslant r$ we add to $\mathcal{B}_{r, 2 M}^{s}$ all the elements

$$
\llbracket X_{i_{1}}, X_{i_{2}}, \ldots, X_{i_{2 M}} \rrbracket_{2 M}
$$

with the (2M)-tuples $\boldsymbol{i}$ formed according to the following rule:

for every $t=0,1, \ldots, M-1$ we take $i$ such that

$$
\begin{aligned}
& i_{j}=i_{2 M+1-j}=1,2, \ldots, s, \quad j=1,2, \ldots, 2 M-t-1, \quad j \neq t+1, \\
& 1 \leqslant i_{t+1}<i_{2 M-t} \leqslant s,
\end{aligned}
$$

provided that $\sum_{j=1}^{2 M} w_{i_{j}}=r$.

Step 3. For every odd $3 \leqslant m=2 M+1 \leqslant r$ we add to $\mathcal{B}_{r, 2 M+1}^{s}$ each element

$$
\llbracket X_{i_{1}}, X_{i_{2}}, \ldots, X_{i_{2 M+1}} \rrbracket_{2 M+1}
$$

for which the $(2 M+1)$-tuple $i$ is either of the form

$$
\begin{aligned}
& i_{j}=i_{2 M+2-j}=1,2, \ldots, s, \quad j=1,2, \ldots, 2 M-t, \quad j \neq t+1, \\
& 1 \leqslant i_{t+1}<i_{2 M+1-t} \leqslant s
\end{aligned}
$$

for some $t \in\{0,1, \ldots, M-1\}$ or

$$
i_{j}=i_{2 M+2-j}=1,2, \ldots, s, \quad j=1,2, \ldots, M, \quad i_{M+1}=1,2, \ldots, s
$$

and, in either case, $\sum_{j=1}^{2 M+1} w_{i_{j}}=r$.

The algorithm above is wasteful, since it forms the same $m$-tuples time and again, and discards the great majority of them since they do not lead to elements in $\mathcal{K}_{r}^{s}(\boldsymbol{w})$. An alternative algorithm, which avoids this pitfall and forms each $m$-tuple just once, is ideally suited to generate the bases $\mathcal{B}_{r}^{s}$ for all $r=1,2, \ldots, r^{\star}$, where $r^{\star} \geqslant 1$ is given.

Algorithm 2. Looping through $m$

Step 0. Let $\mathcal{B}_{r}^{s}=\emptyset, r=1,2, \ldots, r^{\star}$.

Step 1. For every $l=1,2, \ldots, s$, provided that $w_{l} \leqslant r^{\star}$, add $X_{l}$ to $\mathcal{B}_{w_{l}}^{s}$.

Step 2. For every even $m=2 M \leqslant r^{\star}$,

$$
\begin{aligned}
& t=0,1, \ldots, M-1, \\
& i_{1}, i_{2}, \ldots, i_{t}, i_{t+2}, \ldots, i_{2 M-t-1}=1,2, \ldots, s, \\
& 1 \leqslant i_{t+1}<i_{2 M-t} \leqslant s
\end{aligned}
$$




\section{Graded Lie algebras}

evaluate

$$
\rho:=2 \sum_{j=1}^{t} w_{i_{j}}+\sum_{j=t+1}^{2 M-t} w_{i_{j}} .
$$

If $\rho \leqslant r^{\star}$, let $i_{2 M+1-j}=i_{j}$, for $j=1,2, \ldots, t$, and place $\llbracket X_{i_{1}}, X_{i_{2}}, \ldots, X_{i_{2 M}} \rrbracket_{2 M}$ in $\mathcal{B}_{\rho}^{s}$.

Step 3. For every $m=2 M+1 \leqslant r^{\star}, M \geqslant 1$,

$$
\begin{aligned}
& t=0,1, \ldots, M-1, \\
& i_{1}, i_{2}, \ldots, i_{t}, i_{t+2} \ldots, i_{2 M-t}=1,2, \ldots, s, \\
& 1 \leqslant i_{t+1}<i_{2 M+1-t} \leqslant s
\end{aligned}
$$

evaluate

$$
\rho:=2 \sum_{j=1}^{t} w_{i_{j}}+\sum_{j=t+1}^{2 m+1-t} w_{i_{j}} .
$$

Provided that $\rho \leqslant r^{\star}$, let $i_{2 M+2-j}=i_{j}$, for $j=1,2, \ldots, t$, and place the term $\llbracket X_{i_{1}}, X_{i_{2}}, \ldots, X_{i_{2 M+1}} \rrbracket_{2 M+1}$ in $\mathcal{B}_{\rho}^{s}$.

Likewise, for every

$$
i_{1}, i_{2}, \ldots, i_{M+1}=1,2, \ldots, s,
$$

if

$$
\rho:=2 \sum_{j=1}^{M} w_{i_{j}}+w_{i_{M+1}}<r^{\star}
$$

then let $i_{2 M+2-j}=i_{j}, j=1,2, \ldots, M$, and place $\left[X_{i_{1}}, X_{i_{2}}, \ldots, X_{i_{2 M+1}} \rrbracket_{2 M+1}\right.$ in the set $\mathcal{B}_{\rho}^{s}$.

The output of Algorithm 2 consists of the bases $\mathcal{B}_{r}^{s}$ for $r=1,2, \ldots, r^{\star}$. We illustrate it by the example $s=3$ and $w=[1,2,3]$. Letting $r^{\star}=5$, we commence by setting $\mathcal{B}_{r}^{3}=\emptyset$, where $r=1,2, \ldots, 5$. Next, we execute the following steps.

$m=1: X_{1} \rightarrow \mathcal{B}_{1}^{3}, X_{2} \rightarrow \mathcal{B}_{2}^{3}, X_{3} \rightarrow \mathcal{B}_{3}^{3}$.

$m=2$ : The only possibility is $t=0$, and this corresponds to terms $\llbracket X_{i_{1}}, X_{i_{2}} \rrbracket_{2}$. Since $1 \leqslant i_{1}<i_{2} \leqslant 3$, we obtain

$$
\llbracket X_{1}, X_{2} \rrbracket_{2} \rightarrow \mathcal{B}_{3}^{3}, \quad \llbracket\left[X_{1}, X_{3} \rrbracket_{2} \rightarrow \mathcal{B}_{4}^{3}, \quad \llbracket X_{2}, X_{3} \rrbracket_{2} \rightarrow \mathcal{B}_{5}^{3} .\right.
$$

$m=3$ : Again, the only possible value is $t=0$, corresponding to $\left[\left[X_{i_{1}}, X_{i_{2}}, X_{i_{3}} \rrbracket_{3}\right.\right.$ with $i_{2}=1,2,3$ and $1 \leqslant i_{1}<i_{3} \leqslant 3$. Retaining just elements of grade $\leqslant 5$, we have

$$
\llbracket X_{1}, X_{1}, X_{2} \rrbracket_{3} \rightarrow \mathcal{B}_{4}^{3}, \quad \llbracket X_{1}, X_{2}, X_{2} \rrbracket_{3} \rightarrow \mathcal{B}_{5}^{3}, \quad \llbracket X_{1}, X_{1}, X_{3} \rrbracket_{3} \rightarrow \mathcal{B}_{5}^{3} .
$$

The remaining case is $\llbracket X_{i_{1}}, X_{i_{2}}, X_{i_{1}} \rrbracket_{3}$, whence $i_{1}, i_{2}=1,2,3$. We have

$$
\begin{aligned}
& \llbracket\left[X_{1}, X_{1}, X_{1} \rrbracket_{3} \rightarrow \mathcal{B}_{3}^{3}, \quad \llbracket X_{2}, X_{1}, X_{2} \rrbracket_{3} \rightarrow \mathcal{B}_{5}^{3}, \quad \llbracket X_{1}, X_{2}, X_{1} \rrbracket_{3} \rightarrow \mathcal{B}_{4}^{3},\right. \\
& \llbracket X_{1}, X_{3}, X_{1} \rrbracket_{3} \rightarrow \mathcal{B}_{5}^{3} .
\end{aligned}
$$


$m=4$ : We need to consider $t=0$ and $t=1$. In the first case the terms have the form $\llbracket X_{i_{1}}, X_{i_{2}}, X_{i_{3}}, X_{i_{4}} \rrbracket_{4}$ with $i_{2}, i_{3}=1,2,3$ and $1 \leqslant i_{1}<i_{4} \leqslant 3$. There exists just one such term of grade less than or equal to 5 , namely

$$
\llbracket X_{1}, X_{1}, X_{1}, X_{2} \rrbracket_{4} \rightarrow \mathcal{B}_{5}^{3} .
$$

For $t=1$ our terms have the form $\llbracket X_{i_{1}}, X_{i_{2}}, X_{i_{3}}, X_{i_{1}} \rrbracket_{4}$ with $i_{1}=1,2,3$ and $1 \leqslant i_{2}<i_{3} \leqslant 3$. Again, just one term survives,

$$
\llbracket X_{1}, X_{1}, X_{2}, X_{1} \rrbracket_{4} \rightarrow \mathcal{B}_{5}^{3} .
$$

$m=5:$ For $t=0$ we have terms of the form $\llbracket X_{i_{1}}, X_{i_{2}}, X_{i_{3}}, X_{i_{4}}, X_{i_{5}} \rrbracket_{5}$ for $i_{2}, i_{3}, i_{4}=$ $1,2,3$ and $1 \leqslant i_{1}<i_{5} \leqslant 3$. No term of this form has grade less than or equal to 5 . A similar situation pertains to the case $t=1$; the terms are $\left[X_{i_{1}}, X_{i_{2}}, X_{i_{3}}, X_{i_{4}}, X_{i_{1}} \rrbracket_{5}\right.$ with $i_{1}, i_{3}=1,2,3$ and $1 \leqslant i_{2}<i_{4} \leqslant 3$. The remaining case results in the term [ $X_{i_{1}}, X_{i_{2}}, X_{i_{3}}, X_{i_{2}}, X_{i_{1}} \rrbracket_{5}$ with $i_{1}, i_{2}, i_{3}=1,2,3$. One such term is of grade less than or equal to 5 , namely

$$
\llbracket X_{1}, X_{1}, X_{1}, X_{1}, X_{1} \rrbracket_{5} \rightarrow \mathcal{B}_{5}^{3} .
$$

The outcome is the bases

$\mathcal{B}_{1}^{3}=\left\{X_{1}\right\}$;

$\mathcal{B}_{2}^{3}=\left\{X_{2}\right\}$

$\mathcal{B}_{3}^{3}=\left\{X_{3}, \llbracket X_{1}, X_{2} \rrbracket_{2}, \llbracket\left[X_{1}, X_{1}, X_{1} \rrbracket_{3}\right\} ;\right.$

$\mathcal{B}_{4}^{3}=\left\{\llbracket X_{1}, X_{3} \rrbracket_{2}, \llbracket X_{1}, X_{1}, X_{2} \rrbracket_{3}, \llbracket X_{1}, X_{2}, X_{1} \rrbracket_{3}\right\} ;$

$\mathcal{B}_{5}^{3}=\left\{\llbracket X_{2}, X_{3} \rrbracket_{2}, \llbracket X_{1}, X_{2}, X_{2} \rrbracket_{3}, \llbracket X_{1}, X_{1}, X_{3} \rrbracket_{3}, \llbracket X_{2}, X_{1}, X_{2} \rrbracket_{3}\right.$,

$\llbracket X_{1}, X_{3}, X_{1} \rrbracket_{3}, \llbracket\left[X_{1}, X_{1}, X_{1}, X_{2} \rrbracket_{4}, \llbracket\left[X_{1}, X_{1}, X_{2}, X_{1} \rrbracket_{4}, \llbracket\left[X_{1}, X_{1}, X_{1}, X_{1}, X_{1} \rrbracket_{5}\right\}\right.\right.$.

\section{Applications to Cayley-transform methods}

\subsection{The Cayley-BCH formula}

The purpose of this subsection is to present an application of the formalism of hierarchical algebras to the derivation of the Cayley-BCH formula, a relation which in a Cayleytransform setting corresponds to the famous Baker-Hausdorff-Campbell formula.

Let $X, Y \in \mathfrak{g}$, a quadratic algebra of $\mathfrak{g l}_{n}(\mathbb{R})$. Throughout our analysis, we identify $\mathfrak{g}$ with a free hierarchical algebra. For a given sufficiently small $h>0$ we wish to derive a function $Z(h)$ so that the equation

$$
\operatorname{cay}[Z(h)]=\operatorname{cay}(h X) \operatorname{cay}(h Y)
$$

holds. We refer to equation (39) as the Cayley-BCH formula.

Introducing the inverse function cay ${ }^{-1}$ of the Cayley transform (7),

$$
\text { cay }^{-1} W=2(W-I)(W+I)^{-1},
$$

we deduce that the function

$$
Z(h)=\sum_{k=1}^{\infty} Z_{k} h^{k}
$$




\section{Graded Lie algebras}

complies with the equation

$$
Z(h)=2[\operatorname{cay}(h X) \operatorname{cay}(h Y)-I] \times[\operatorname{cay}(h X) \operatorname{cay}(h Y)+I]^{-1} .
$$

Consequently,

$$
\begin{aligned}
& Z(h)=2\left[\left(I-\frac{1}{2} h X\right)^{-1}\left(I+\frac{1}{2} h X\right)\left(I-\frac{1}{2} h Y\right)^{-1}\left(I+\frac{1}{2} h Y\right)+I\right]^{-1} \\
& \times\left[\left(I-\frac{1}{2} h X\right)^{-1}\left(I+\frac{1}{2} h X\right)\left(I-\frac{1}{2} h Y\right)^{-1}\left(I+\frac{1}{2} h Y\right)-I\right] \\
&= {\left[\left(I-\frac{1}{2} h X\right)^{-1}\left(I+\frac{1}{4} h^{2} X Y\right)\left(I-\frac{1}{2} h Y\right)^{-1}\right]^{-1} } \\
& \times\left[\left(I-\frac{1}{2} h X\right)^{-1}(X+Y)\left(I-\frac{1}{2} h Y\right)^{-1}\right] \\
&= h\left(I-\frac{1}{2} h Y\right)\left(I+\frac{1}{4} h^{2} X Y\right)^{-1}(X+Y)\left(I-\frac{1}{2} h Y\right)^{-1} .
\end{aligned}
$$

Multiplying both sides on the left by $\left(I-\frac{1}{2} h Y\right)$ and writing $\left(I+\frac{1}{4} h^{2} X Y\right)^{-1}$ as a geometric series, we infer that

$$
Z(h)\left(I-\frac{1}{2} h Y\right)=\left(I-\frac{1}{2} h Y\right)\left(\sum_{k=0}^{\infty} \frac{(-1)^{k}}{2^{2 k}}(X Y)^{k} h^{2 k+1}\right)(X+Y),
$$

hence the recursions

$$
\begin{aligned}
& Z_{2 k+1}=\frac{1}{2} Z_{2 k} Y+\frac{(-1)^{k}}{2^{2 k}}(X Y)^{k}(X+Y), \\
& Z_{2 k+2}=\frac{1}{2} Z_{2 k+1} Y-\frac{(-1)^{k}}{2^{2 k+1}} Y(X Y)^{k}(X+Y) .
\end{aligned}
$$

(with starting condition $Z_{0}=O$ ), that determine the $Z_{k}$.

Theorem 8. With the same notation as above, it is true that

$$
\begin{aligned}
Z_{2 k+1} & =\frac{(-1)^{k}}{2^{2 k+1}}\left(\llbracket X, Y, \ldots, X, Y, X \rrbracket_{2 k+1}+\llbracket Y, X, \ldots Y, X, Y \rrbracket_{2 k+1}\right), \\
Z_{2 k+2} & =\frac{(-1)^{k}}{2^{2 k+1}} \llbracket X, Y, \ldots, X, Y \rrbracket_{2 k+2},
\end{aligned}
$$

for $k=0,1,2, \ldots$.

Proof. This is achieved by induction on $k$. For $k=0$ we obtain

$$
\begin{aligned}
& Z_{1}=\frac{1}{2}\left(\llbracket X \rrbracket_{1}+\llbracket Y \rrbracket_{1}\right)=X+Y, \\
& Z_{2}=\frac{1}{2} \llbracket X, Y \rrbracket_{2}=X Y-Y X,
\end{aligned}
$$

which is clearly in agreement with the recursions (40). Next, let us assume that the assertion is true for all integers up to $k$. We deduce from the recurrence relations (40) that

$$
\begin{aligned}
Z_{2 k+1} & =\frac{1}{2} Z_{2 k} Y+\frac{(-1)^{k}}{2^{2 k}}(X Y)^{k}(X+Y) \\
& =\frac{(-1)^{k-1}}{2^{2 k}}\left(\llbracket X, Y, \ldots, X, Y \rrbracket_{2 k} Y-(X Y)^{k}(X+Y)\right)
\end{aligned}
$$




\section{Graded Lie algebras}

$$
\begin{aligned}
& =\frac{(-1)^{k}}{2^{2 k}}(X Y \cdots Y X+Y X \cdots X Y) \\
& =\frac{(-1)^{k}}{2^{2 k+1}}\left(\llbracket X, Y, \ldots, Y, X \rrbracket_{2 k+1}+\llbracket Y, X, \ldots, X, Y \rrbracket_{2 k+1}\right) .
\end{aligned}
$$

By a similar token,

$$
\begin{aligned}
Z_{2 k+2}= & \frac{1}{2} Z_{2 k+1} Y-\frac{(-1)^{k}}{2^{2 k+1}} Y(X Y)^{k}(X+Y) \\
= & \frac{(-1)^{k}}{2^{2 k+2}}\left(\llbracket X, Y, \ldots, Y, X \rrbracket_{2 k+1}+\llbracket Y, X, \ldots, X, Y \rrbracket_{2 k+1}\right) \\
& \quad-\frac{(-1)^{k}}{2^{2 k+1}} Y(X Y)^{k}(X+Y) \\
= & \frac{(-1)^{k}}{2^{2 k+1}}(X Y X \cdots Y X Y+Y X Y \cdots X Y Y \\
& \quad-Y X Y \cdots X Y X-Y X Y \cdots X Y Y) \\
= & \frac{(-1)^{k}}{2^{2 k+1}} \llbracket X, Y, X, \ldots, Y, X \rrbracket_{2 k+2},
\end{aligned}
$$

and hence the assertion is true also for $k+1$. The proof is complete.

In passing, it is worthwhile to mention that, in variance from the case of the exponential mapping, whereas

$$
\exp (h X) \exp (h X)=\exp (2 h X)
$$

one has

$$
\operatorname{cay}(h X) \operatorname{cay}(h X)=\operatorname{cay}(Z(h)),
$$

for the Cayley map, where

$$
Z(h)=\sum_{k=0}^{\infty} Z_{2 k+1} h^{2 k+1}=\frac{4}{\mathrm{i}} \sum_{k=0}^{\infty}\left(\frac{\mathrm{i} h X}{2}\right)^{2 k+1}=2 h X\left(I+\frac{h^{2} X^{2}}{4}\right)^{-1} .
$$

\subsection{Adjoint operators in a Cayley-transform setting}

As above, we consider two generators, say $X$ and $Y$ in $\mathfrak{g}$. We wish to derive a formula for the hierarchical-algebra element

$$
\operatorname{cay}(h X) Y \operatorname{cay}(-h X)
$$

in terms of primitive elements of a hierarchical algebra. To this end, we observe that

$$
\operatorname{cay}(V)=I+2 \sum_{k=1}^{\infty}\left(\frac{h}{2}\right)^{k} V^{k}, \quad V \in \mathfrak{g} ;
$$

hence we obtain by direct computation

$$
\begin{aligned}
\operatorname{cay}(h X) Y \operatorname{cay}(-h X)=Y & +2 \sum_{k=1}^{\infty}\left(\frac{h}{2}\right)^{k}\left[X^{k} Y+(-1)^{k} Y X^{k}\right] \\
& +4 \sum_{k=2}^{\infty}\left(\frac{h}{2}\right)^{k} \sum_{j=1}^{k-1}(-1)^{k-j} X^{j} Y X^{k-j},
\end{aligned}
$$




\section{Graded Lie algebras}

which, using hierarchical-algebra primitive elements, translates to

$$
\begin{aligned}
\operatorname{cay}(h X) Y \operatorname{cay}(-h X)= & \frac{1}{2} \llbracket Y \rrbracket_{1}+2 \sum_{k=1}^{\infty}\left(\frac{h}{2}\right)^{k} \llbracket X, \ldots, X, Y \rrbracket_{k+1} \\
& +4 \sum_{k=2}^{\infty}\left(\frac{h}{2}\right)^{k} \sum_{j=1}^{\left\lfloor\frac{k}{2}\right\rfloor}(-1)^{k-j} \alpha_{j} \llbracket \underbrace{X, \ldots, X}_{j}, Y, \underbrace{X, \ldots, X}_{k-j} \rrbracket_{k+1},
\end{aligned}
$$

where $\alpha_{1}=\cdots=\alpha_{\lfloor(k-1) / 2\rfloor}=1$ and $\alpha_{k / 2}=1 / 2$ when $k$ is an even index. Hence, for each $k$, the terms are precisely the primitive elements of length $k$ with a single occurrence of $Y$.

Assume that $w(X)=w_{X}$ and $w(Y)=w_{Y}$. Then, the grade of a primitive word of length $k$ and $k-1$ occurrences of $X$ is $(k-1) w_{X}+w_{Y}$; therefore, identifying grades with order of accuracy, in order to truncate the above formula to an order $K$, we require that the above summation be carried up to the least index $\hat{k}$ such that

$$
\frac{K+w_{X}-w_{Y}}{w_{X}} \leqslant \hat{k}
$$

holds.

We have mentioned above that the adjoint operator in a Cayley setting is a sum of primitive elements of the hierarchical algebra, with a single occurrence of $Y$. In greater generality, denote by $\gamma_{R ; l}$ the number of primitive words of length $R$ and $l$ occurrences of the letter $X$. Clearly,

$$
\gamma_{R ; l}=\gamma_{R ; R-l}, \quad l=0,1, \ldots, R
$$

and, furthermore,

$$
d_{R}^{2}=\sum_{l=0}^{R} \gamma_{R ; l},
$$

where $d_{R}^{2}$ is the dimension of the space $\mathcal{K}_{R}^{2}(\mathbf{1})$.

Lemma 9. With the same notation as above, it is true that

$$
\begin{array}{rlrl}
\gamma_{2 R+1 ; 2 l} & =\gamma_{2 R ; 2 l-1}+\gamma_{2 R ; 2 l}+\left(\begin{array}{c}
R \\
l
\end{array}\right), & l & =0, \ldots, R, \\
\gamma_{2 R+1 ; 2 l+1}=\gamma_{2 R ; 2 l}+\gamma_{2 R ; 2 l+1}+\left(\begin{array}{c}
R \\
l
\end{array}\right), & l=0, \ldots, R, \\
\gamma_{2 R+2 ; 2 l}=\gamma_{2 R+1 ; 2 l-1}+\gamma_{2 R+1 ; 2 l}-\left(\begin{array}{c}
R+1 \\
l
\end{array}\right), & l=0, \ldots, R+1, \\
\gamma_{2 R+2 ; 2 l+1}=\gamma_{2 R+1 ; 2 l}+\gamma_{2 R+1 ; 2 l+1}-\left(\begin{array}{c}
R \\
l
\end{array}\right), & l=0, \ldots, R .
\end{array}
$$

Proof. We proceed by induction on the length of the primitive word. Let us consider the index $R=0$ and words of length one. The only possible primitive words are $[X]_{1}$ and [[Y $Y]_{1}$; hence $\gamma_{1 ; 0}=1$ and $\gamma_{1 ; 1}=1$, which is in accordance with equations (41) and (42) respectively, given that $\gamma_{0 ; l}=0$. For words of length 2 , we observe that $\gamma_{2 ; 0}=0=\gamma_{2 ; 2}$, since there are no $\left[\because Y, Y \rrbracket_{2}\right.$ and $\llbracket X, X \rrbracket_{2}$ words. Instead, $\gamma_{2 ; 1}=1$, corresponding to $\llbracket X, Y \rrbracket_{2}$, and equations (43) and (44) are obeyed. 


\section{Graded Lie algebras}

Next, let us assume that the result is true for $k=1,2, \ldots, 2 R$. If $\llbracket X, Y \ldots, Y, X \rrbracket_{2 R}$ is a primitive word of length $2 R$ with $L$ occurrences of $X$, then $\llbracket X, Y, \ldots, Y, X, Y \rrbracket_{2 R+1}$, obtained by appending a $Y$, is a primitive word of length $2 R+1$ with $L$ occurrences of $X$. Similarly, we can take a primitive word of length $2 R$ with $L-1$ occurrences of $X$, and obtain one of length $2 R+1$ with $L$ occurrences of $X$ by appending an $X$ to it. However, by doing so, we do not obtain all the primitive words, because there are no symmetric words of length $2 R$. All the missing words can be obtained by choosing $R$ slots (for instance the first $R$ ) and letting $\lfloor L / 2\rfloor$ letters $X$ occur in them. Hence

$$
\gamma_{2 R+1 ; L}=\gamma_{2 R ; L-1}+\gamma_{2 R, L}+\left(\begin{array}{c}
R \\
\lfloor L / 2\rfloor
\end{array}\right),
$$

which gives equations (41) and (42). A similar procedure applies for equations (43) and (44), except that in this case we have to remove symmetric words which do not appear in the set of primitive elements.

In other words, the number of words with a given number of occurrences of $X$ and $Y$ obeys a generalized version of the binomial coefficient relation. Table 2 shows the values of $\gamma_{R ; l}$.

Table 2: A tabulation of $\gamma_{R ; l}$

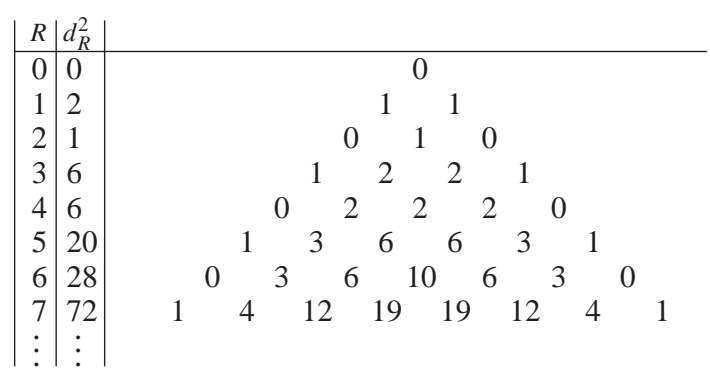

Each entry can be obtained as the sum of the two entries directly above, plus the corresponding entry of the alternating Pascal triangle in Table 3.

Table 3: The alternating Pascal triangle

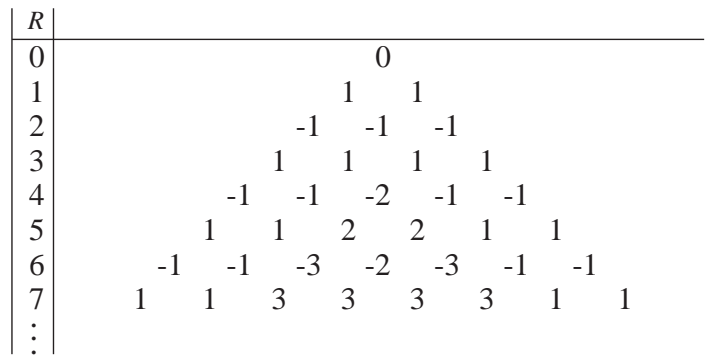




\section{Graded Lie algebras}

Theorem 10. The generating function

$$
\Gamma(x, y)=\sum_{R=0}^{\infty} \sum_{l=0}^{R} \gamma_{R ; l} x^{R-l} y^{l}
$$

for the coefficients $\gamma_{R ; l}$ is

$$
\Gamma(x, y)=\frac{x+y-x y-\left(x^{2}+y^{2}\right)}{(1-x-y)\left(1-x^{2}-y^{2}\right)} .
$$

Proof. Letting $\gamma_{R ; l}=0$ for $l>R$, we extend the range of second summation to $l \geqslant 0$. We have

$$
\begin{aligned}
\Gamma(x, y) & =\sum_{R=0}^{\infty} \gamma_{R ; 0} x^{R}+\sum_{R=1}^{\infty} \sum_{l=1}^{\infty} \gamma_{R ; l} x^{R-l} y^{l} \\
& =\frac{x}{1-x^{2}}+\sum_{R=1}^{\infty} \sum_{l=1}^{\infty} \gamma_{R ; l} x^{R-l} y^{l},
\end{aligned}
$$

since $\gamma_{2 k, 0}=0$ and $\gamma_{2 k+1 ; 0}=1, k=0,1,2, \ldots$ Next, we rearrange the second sum separating even and odd values of the first and of the second index and use Lemma 9 to obtain

$$
\begin{aligned}
\Gamma(x, y)=\frac{x}{1-} x^{2} & +\sum_{R=0}^{\infty} \sum_{l=0}^{\infty} \gamma_{2 R+1 ; 2 l+1} x^{2(R-l)} y^{2 l+1} \\
& +\sum_{R=0}^{\infty} \sum_{l=0}^{\infty} \gamma_{2 R+1 ; 2 l+2} x^{2(R-l)-1} y^{2 l+2} \\
& +\sum_{R=0}^{\infty} \sum_{l=0}^{\infty} \gamma_{2 R+2 ; 2 l+1} x^{2(R-l)+1} y^{2 l+1} \\
& +\sum_{R=0}^{\infty} \sum_{l=0}^{\infty} \gamma_{2 R+2 ; 2 l+2} x^{2(R-l)} y^{2 l+2} \\
= & x \quad+\sum^{2} \sum_{l=0}^{\infty}\left[\gamma_{2 R ; 2 l+1}+\gamma_{2 R ; 2 l}+\left(\begin{array}{c}
R \\
l
\end{array}\right)\right] x^{2(R-l)} y^{2 l+1} \\
& +\sum_{R=0}^{\infty} \sum_{l=0}^{\infty}\left[\gamma_{2 R ; 2 l+2}+\gamma_{2 R ; 2 l+1}+\left(\begin{array}{c}
R \\
l+1
\end{array}\right)\right] x^{2(R-l)-1} y^{2 l+2} \\
& +\sum_{R=0}^{\infty} \sum_{l=0}^{\infty}\left[\gamma_{2 R+1 ; 2 l+1}+\gamma_{2 R+1 ; 2 l}-\left(\begin{array}{c}
R \\
l
\end{array}\right)\right] x^{2(R-l)+1} y^{2 l+1} \\
& +\sum_{R=0}^{\infty} \sum_{l=0}^{\infty}\left[\gamma_{2 R+1 ; 2 l+2}+\gamma_{2 R+1 ; 2 l+1}-\left(\begin{array}{c}
R+1 \\
l+1
\end{array}\right)\right] x^{2(R-l)} y^{2 l+2} .
\end{aligned}
$$

Note that

$$
\sum_{R=0}^{\infty} \sum_{l=0}^{\infty}\left[\gamma_{2 R ; 2 l} x^{2(R-l)} y^{2 l+1}+\gamma_{2 R ; 2 l+1} x^{2(R-l)-1} y^{2 l+2}\right.
$$




\section{Graded Lie algebras}

$$
\left.+\gamma_{2 R+1 ; 2 l} x^{2(R-l)+1} y^{2 l+1}+\gamma_{2 R+1 ; 2 l+1} x^{2(R-l)} y^{2 l+2}\right]=y \Gamma(x, y),
$$

and that

$$
\begin{aligned}
& \sum_{R=0}^{\infty} \sum_{l=0}^{\infty}\left[\gamma_{2 R ; 2 l+1} x^{2(R-l)} y^{2 l+1}+\gamma_{2 R ; 2 l+2} x^{2(R-l)-1} y^{2 l+2}\right. \\
& \left.\quad+\gamma_{2 R+1 ; 2 l+1} x^{2(R-l)+1} y^{2 l+1}+\gamma_{2 R+1 ; 2 l+2} x^{2(R-l)} y^{2 l+2}\right]=x \Gamma(x, y)-\frac{x^{2}}{1-x^{2}} .
\end{aligned}
$$

Furthermore,

$$
\begin{aligned}
\sum_{R=0}^{\infty} \sum_{l=0}^{\infty}\left(\begin{array}{l}
R \\
l
\end{array}\right) x^{2(R-l)} y^{2 l+1} & =y \sum_{R=0}^{\infty}\left(x^{2}+y^{2}\right)^{R}=\frac{y}{1-\left(x^{2}+y^{2}\right)}, \\
\sum_{R=0}^{\infty} \sum_{l=0}^{\infty}\left(\begin{array}{c}
R \\
l+1
\end{array}\right) x^{2(R-l)-1} y^{2 l+2} & =\frac{x}{1-\left(x^{2}+y^{2}\right)}-\frac{x}{1-x^{2}} \\
\sum_{R=0}^{\infty} \sum_{l=0}^{\infty}\left(\begin{array}{l}
R \\
l
\end{array}\right) x^{2(R-l)+1} y^{2 l+1} & =\frac{x y}{1-\left(x^{2}+y^{2}\right)} \\
\sum_{R=0}^{\infty} \sum_{l=0}^{\infty}\left(\begin{array}{l}
R+1 \\
l+1
\end{array}\right) x^{2(R-l)} y^{2 l+2} & =\frac{x^{2}+y^{2}}{1-\left(x^{2}+y^{2}\right)}-\frac{x^{2}}{1-x^{2}} .
\end{aligned}
$$

Subsituting above and collecting terms, we deduce that

$$
(1-x-y) \Gamma(x, y)=\frac{x+y-x y-\left(x^{2}+y^{2}\right)}{1-\left(x^{2}+y^{2}\right)},
$$

and hence the theorem follows.

We note that, setting $q(x, y)=x+y$, the above generating function becomes

$$
\Gamma(x, y)=\frac{q(x, y)-\frac{1}{2} q\left(x^{2}, y^{2}\right)-\frac{1}{2}[q(x, y)]^{2}}{[1-q(x, y)]\left[1-q\left(x^{2}, y^{2}\right)\right]},
$$

a two-variable counterpart of equation (27).

Theorem 11. With the same notation as above, it is true that

$$
\begin{aligned}
\gamma_{2 R ; 2 l} & =\frac{1}{2}\left[\left(\begin{array}{c}
2 R \\
2 l
\end{array}\right)-\left(\begin{array}{l}
R \\
l
\end{array}\right)\right], \\
\gamma_{2 R ; 2 l+1} & =\frac{1}{2}\left(\begin{array}{c}
2 R \\
2 l+1
\end{array}\right), \\
\gamma_{2 R+1 ; 2 l} & =\frac{1}{2}\left[\left(\begin{array}{c}
2 R+1 \\
2 l
\end{array}\right)+\left(\begin{array}{l}
R \\
l
\end{array}\right)\right], \\
\gamma_{2 R+1 ; 2 l+1} & =\frac{1}{2}\left[\left(\begin{array}{c}
2 R+1 \\
2 l+1
\end{array}\right)+\left(\begin{array}{l}
R \\
l
\end{array}\right)\right],
\end{aligned}
$$

for all $R, l \geqslant 0$.

Proof. The generating function $\Gamma(x, y)$ can be written as

$$
\Gamma(x, y)=\frac{1}{2} \frac{1}{1-(x+y)}+\frac{1}{2} \frac{x+y-1}{1-\left(x^{2}+y^{2}\right)} .
$$


We observe that

$$
\frac{1}{1-(x+y)}=\sum_{k=0}^{\infty} \sum_{l=0}^{k}\left(\begin{array}{l}
k \\
l
\end{array}\right) x^{k-l} y^{l}
$$

is the generating function of the binomial coefficients. Similarly,

$$
\frac{1}{1-\left(x^{2}+y^{2}\right)}=\sum_{k=0}^{\infty} \sum_{l=0}^{k}\left(\begin{array}{l}
k \\
l
\end{array}\right) x^{2(k-l)} y^{2 l} .
$$

Multiplying the latter by $x+y-1$ and matching powers yields the desired result.

\subsection{Cayley expansions}

The most important application of the counting results presented in Section 3 is to numerical methods based on a Cayley expansion [11] for the solution of the differential equation (1). Recall that the solution of the dcayinv equation (8) can be written as

$$
\Omega(t)=\sum_{m=1}^{\infty} \mathcal{I}_{m}(t)
$$

where the functions $\mathcal{I}_{m}(t)$ obey the recurrence relation [11]:

$$
\mathcal{I}_{m}(t)=-\frac{1}{2} \int_{0}^{t}\left[\mathcal{I}_{m-1}(\xi), A(\xi)\right] \mathrm{d} \xi-\frac{1}{4} \sum_{k=1}^{m-2} \int_{0}^{t} \mathcal{I}_{m-k-1}(\xi) A(\xi) \mathcal{I}_{k}(\xi) \mathrm{d} \xi, \quad m \geqslant 3,
$$

with starting condition

$$
\mathcal{I}_{1}(t)=\int_{0}^{t} A(\xi) \mathrm{d} \xi
$$

The sum on the right-hand side of equation (45) should be considered null when the range of indices is empty. As observed in Section 1, each $\mathcal{I}_{m}(t)$ features commutators and symmetric products of the form $P Q R+R Q P=\llbracket P, Q, R \rrbracket_{3}$. We invoke the hierarchy condition of the $m$-nary operator and claim that

$$
\mathcal{I}_{m}(h)=\sum_{\boldsymbol{k}} \alpha_{\boldsymbol{k}} \int_{h s} \llbracket\left[A\left(\xi_{k_{1}}\right), \ldots, A\left(\xi_{k_{m}}\right) \rrbracket_{m} \mathrm{~d} \xi_{k_{1}} \cdots \mathrm{d} \xi_{k_{m}},\right.
$$

where the integration is performed over some polytope $\&$. The $m$-nary product is linear in each of its components! Therefore, given quadrature points $c_{1}, \ldots, c_{v}$ in $[0,1]$, and denoting $A_{k}=A\left(c_{k} h\right)$, we can approximate each of the terms

$$
\int_{h \S} \llbracket A\left(\xi_{k_{1}}\right), \ldots, A\left(\xi_{k_{m}}\right) \rrbracket_{m} \mathrm{~d} \xi_{k_{1}} \cdots \mathrm{d} \xi_{k_{m}},
$$

with a quadrature formula

$$
\mathcal{Q}(h)=h^{m} \sum_{k_{1}=1}^{v} \cdots \sum_{k_{m}=1}^{v} b_{\boldsymbol{k}} \llbracket\left[A_{k_{1}}, \ldots, A_{k_{m}} \rrbracket_{m},\right.
$$

the weights $b_{\boldsymbol{k}}$ being obtained by integrating products of cardinal Lagrange polynomials over the polytope $\&$. In such a case all the terms $A_{k_{1}}, \ldots, A_{k_{m}}$ are representations of some free hierarchical algebra symbols $X_{1}, \ldots, X_{m}$, and, for each degree $m$ we are required to 
evaluate a number of terms that is bounded by the dimension of the basis of $\mathcal{K}_{m}^{v}(\mathbf{1})$. As an example, let us consider two Gaussian nodes $c_{1}=\frac{1}{2}-\frac{\sqrt{3}}{6}$ and $c_{2}=\frac{1}{2}+\frac{\sqrt{3}}{6}$ in $[0,1]$, which we can use to generate a method of order four. Taking into account further simplifying assumptions such as time-symmetry, a bound on the number of terms is given by the sum of the dimensions of graded subspaces $\mathcal{K}_{m}^{2}(\mathbf{1})$, for $m=1, \ldots, 3$ (that is, nine; see Table 1). The required primitive terms are

$$
\begin{array}{lllll}
\llbracket A_{1} \rrbracket_{1} & \llbracket A_{2} \rrbracket_{1} & \llbracket A_{1}, A_{2} \rrbracket_{2} & \llbracket A_{1}, A_{1}, A_{1} \rrbracket_{3} & \llbracket A_{1}, A_{1}, A_{2} \rrbracket_{3} \\
\llbracket A_{1}, A_{2}, A_{1} \rrbracket_{3} & \llbracket A_{2}, A_{2}, A_{1} \rrbracket_{3} & \llbracket A_{2}, A_{1}, A_{2} \rrbracket_{3} & \llbracket A_{2}, A_{2}, A_{2} \rrbracket_{3} . &
\end{array}
$$

Considerable savings occur when we choose graded algebras. Assume that $\left\{B_{0}, B_{1}\right\}$ is a base equivalent to $\left\{A_{1}, A_{2}\right\}$, but with weights $w=[1,2]$. Such a basis can be obtained by means of a Vandermonde transformation, as described in Section 1. Since the polynomial

$$
p(z)=z^{2}-z-1
$$

has two distinct roots, namely $\lambda=\frac{1 \pm \sqrt{5}}{2}$, according to Theorem 5 ,

$$
\begin{aligned}
\operatorname{dim} \mathcal{K}_{2 R}^{2}[1,2] & =\frac{1}{2} \sum_{l=1}^{2} \frac{1}{-1+2 \lambda_{l}}\left(\lambda_{l}^{2 R+1}-\lambda_{l}^{R+1}+\lambda_{l}^{R}\right) \\
\operatorname{dim} \mathcal{K}_{2 R+1}^{2}[1,2] & =\frac{1}{2} \sum_{l=1}^{2} \frac{1}{-1+2 \lambda_{l}}\left(\lambda_{l}^{2 R+2}+\lambda_{l}^{R+1}\right),
\end{aligned}
$$

and we deduce that $\operatorname{dim} \mathcal{K}_{1}^{2}(\boldsymbol{w})=\operatorname{dim} \mathcal{K}_{2}^{2}(\boldsymbol{w})=1$ and $\operatorname{dim} \mathcal{K}_{3}^{2}(\boldsymbol{w})=2$, yielding a total of four terms, corresponding to

$$
\llbracket B_{0} \rrbracket_{1}, \quad \llbracket B_{1} \rrbracket_{1}, \quad \llbracket\left[B_{0}, B_{1} \rrbracket_{2}, \quad \llbracket\left[B_{0}, B_{0}, B_{0} \rrbracket_{3} .\right.\right.
$$

\subsection{Runge-Kutta-Munthe-Kaas schemes in a Cayley-transform setting}

As a last example, we will consider the application of our results to Runge-KuttaMunthe-Kaas methods when employing the Cayley transform as a map from the Lie algebra to the corresponding Lie group. For the more general setting of homogeneous manifolds, we refer the reader to [7].

The numerical methods in question consist of solving the differential equation (1) by obtaining a numerical solution to the dcayinv equation (8) in $\mathfrak{g}$ by means of a Runge-Kutta method, and mapping back the result to the Lie group $G$ by means of the Cayley transform (7). Given a $v$-stage Runge-Kutta scheme, defined in terms of the tableau

$$
\begin{array}{c|cccc}
c_{1} & a_{1,1} & a_{1,2} & \cdots & a_{1, v} \\
\vdots & \vdots & \vdots & & \vdots \\
c_{v} & a_{\nu, 1} & a_{v, 2} & \cdots & a_{v, v} \\
\hline & b_{1} & b_{2} & \cdots & b_{v}
\end{array}
$$

to advance the solution by a single step from $t_{N}$ to $t_{N+1}=t_{N}+h$ we compute

$$
\begin{aligned}
\Phi_{m} & =h \sum_{l=1}^{v} a_{m, l} \tilde{K}_{l}, \\
A_{m} & =A\left(t_{N}+h c_{m}\right), \quad m=1,2, \ldots, v ; \\
K_{m} & =\operatorname{dcay}_{\Phi_{i}}^{-1}\left(A_{m}\right),
\end{aligned}
$$


hence,

$$
\Omega_{n+1}=h \sum_{m=1}^{v} b_{m} K_{m}
$$

and, finally,

$$
Y_{N+1}=\operatorname{cay}\left(h \Omega_{N+1}\right) Y_{N} .
$$

The matrices $\tilde{A}_{1}, \ldots, \tilde{A}_{v}$ are a representation of generators $X_{1}, \ldots, X_{v}$ of the free hierarchical algebra, with grades $w\left(X_{i}\right)=1$, for $i=1,2, \ldots, v$. Thus, an expansion in primitive words requires

$$
\sum_{m=1}^{p} \operatorname{card}\left\{W \in \mathcal{B}_{m}^{v}(\mathbf{1}) \mid W \text { has length } \leqslant 3\right\}
$$

terms, where $p \leqslant 2 v$ is the order of the numerical method in question. Again, one can use a graded algebra generated by $B_{0}, \ldots, B_{v-1}$ with grades $w=[1,2, \ldots, v]$, and reduce the number of required primitive words to

$$
\sum_{m=1}^{p} \operatorname{card}\left\{W \in \mathcal{B}_{m}^{v}(\boldsymbol{w}) \mid W \text { has length } \leqslant 3\right\} .
$$

The savings occur by virtue of the fact that usually $\mathcal{B}_{m}^{v}(\boldsymbol{w})$ has a cardinality substantially smaller than $\mathcal{B}_{m}^{v}(\mathbf{1})$.

We should mention that for this particular example, it is not necessarily true that the introduction of primitive words would lead to a significantly cheaper computation, due to the fact that, unlike the dexp ${ }^{-1}$ equation, the dcay ${ }^{-1}$ formula is finite and consists of a very small number of terms.

Acknowledgements. The authors wish to thank members of the Numerical Analysis group at DAMTP, University of Cambridge, for numerous discussions and comments, and for allowing us to share our enthusiasm for hierarchical algebras. Particular thanks are due to Malcolm Sabin.

\section{References}

1. C. J. BudD and A. IsERles, 'Geometric integration: numerical solution of differential equations on manifolds', Philos. Trans. Roy. Soc. London Ser. A 357 (1999) 945-956. 44,45

2. E. Celledoni and A. Iserles, 'Methods for the approximation of the matrix exponential in a Lie-algebraic setting', Tech. Rep. 015, Mathematical Sciences Research Institute, Berkeley, California, 1999; IMA J. Numer. Anal., to appear. 45

3. R. Cools, 'Constructing cubature formulae: the science behind the art', Acta Numerica 6 (1997) 1-54. 48

4. P. CRouch and R. Grossman, 'Numerical integration of ordinary differential equations on manifolds', J. Nonlinear Sci. 3 (1993) 1-33. 45

5. L. DieCi, R. D. RusSEll and E. S. VAN VLECK, 'Unitary integrators and applications to continuous orthonormalization techniques', SIAM J. Numer. Anal. 31 (1994) 261-281. 45

6. F. Diele, L. Lopez and R. Peluso, 'The Cayley transform in the numerical solution of unitary differential systems', Adv. Comput. Math. 8 (1998) 317-334. 45, 47 
7. K. EnGø, 'On the construction of geometric integrators in the RKMK class', Tech. Rep. No. 158, Department of Informatics, University of Bergen, Norway, 1998. 45, 47, 73

8. F. Fer, 'Résolution del l'equation matricielle $\dot{U}=p U$ par produit infini d'exponentielles matricielles', Acad. Roy. Belg. Bull. Cl. Sci. 44 (1958) 818-829. 46

9. A. ISERLES, 'Solving linear ordinary differential equations by exponentials of iterated commutators', Numer. Math. 45 (1984) 183-199. 46

10. A. IsERLes, A first course in the numerical analysis of differential equations (Cambridge University Press, Cambridge, 1996). 46

11. A. IsERLES, 'On Cayley-transform methods for the discretization of Lie-group equations', Tech. Rep. NA1999/4, Department of Applied Mathematics and Theoretical Physics, University of Cambridge, 1999. 45, 45, 47, 47, 47, 48, 72, 72

12. A. IsERLES and S. P. NøRSETT, 'On the solution of linear differential equations in Lie groups', Philos. Trans. Roy. Soc. London Ser. A 357 (1999) 983-1019. 46, 48, 48, 48

13. D. Lewis and J. C. Simo, 'Conserving algorithms for the dynamics of Hamiltonian systems on Lie groups', J. Nonlinear Sci. 4 (1994) 253-299. 45, 47

14. W. MaGnus, 'On the exponential solution of differential equations for a linear operator', Comm. Pure Appl. Maths VII (1954) 649-673. 46

15. R. I. McLachlan, G. R. W. Quispel and N. Robidoux, 'Geometric integration using discrete gradients', Philos. Trans. Roy. Soc. London Ser. A 357 (1999) 1021-1045. 45

16. H. Munthe-KaAs, 'Runge-Kutta methods on Lie groups', BIT 38 (1998) 92-111. 45

17. H. Munthe-Kans and B. Owren, 'Computations in a free Lie algebra', Philos. Trans. Roy. Soc. London Ser. A 357 (1999) 957-981. 45, 45, 45, 45, 48, 48, 49, 49, 49, 52, $54,54,63$

18. H. Munthe-KaAs and A. Zanna, 'Numerical integration of differential equations on homogeneous manifolds', Foundations of computational mathematics (ed. F. Cucker and M. Shub, Springer Verlag, 1997) 305-315. 45

19. A. L. ONischiK (ed.), Lie groups and Lie algebras, Encyclopaedia Math. Sci. 20 (Springer-Verlag, Berlin, 1993). 54

20. B. OWren and A. Marthinsen, 'Integration methods based on canonical coordinates of the second kind', Tech. Rep. Numerics No. 5/1999, Norwegian University of Science and Technology, Trondheim, Norway, 1999. 45

21. A. Zanna, 'Collocation and relaxed collocation for the Fer and the Magnus expansions', Tech. Rep. NA1997/17, Department of Applied Mathematics and Theoretical Physics, University of Cambridge, 1997; SIAM J. Num. Anal., to appear. 46, 46

Arieh Iserles A.Iserles@damtp.cam.ac.uk

Antonella Zanna A.Zanna@damtp.cam.ac.uk

Department of Applied Mathematics and Theoretical Physics

University of Cambridge

Silver Street

Cambridge CB3 9EW 